\title{
MINIMAL BIT RATES AND ENTROPY FOR EXPONENTIAL STABILIZATION*
}

\author{
FRITZ COLONIUS ${ }^{\dagger}$
}

\begin{abstract}
Minimal bit rates and entropy are studied for exponential stabilization of control systems in continuous time. Upper and lower bounds for the stabilization entropy are derived. In particular, for linear systems, a formula is given in terms of the real parts of eigenvalues. Then the minimal bit rate is related to the stabilization entropy.
\end{abstract}

Key words. stabilization entropy, minimal bit rates

AMS subject classifications. 93D15, 94A17

DOI. $10.1137 / 110829271$

1. Introduction. The problem to determine minimal data rates for performing control tasks has been considered for more than 20 years; see the survey by Nair et al. [16]. Early landmarks are the papers by Delchamps [4], who considered quantized information for stabilization and proposed to use statistical methods from ergodic theory, and Wong and Brockett [22], who discussed stabilization of linear systems via coding. From the wealth of literature on this topic we also cite Tatikonda and Mitter [20], Delvenne [5], Fagnani and Zampieri [8], Matveev and Savkin [14], Nair et al. [17], and Xie [23]. The present paper proposes an approach that is different from quantization of the state space in order to analyze stabilization about an equilibrium. In particular, no information pattern is specified describing how the information on the states of the system is available for the controller. The approach is based on ideas from [17], where for discrete-time systems the notion of topological feedback entropy is introduced which has similarities to the notion of topological entropy in the theory of dynamical systems as presented in Katok and Hasselblatt [10] or Walters [21], and on concepts from Colonius and Kawan [2] and Kawan [11, 12, 13], where invariance entropy for controlled invariance has been studied.

Taking up an idea formulated in Tatikonda and Mitter [20, p. 1057], we consider the time dependent controls which have to be generated by a controller in order to achieve exponential stability. In contrast to most of the literature, we consider continuous time systems. There are two ways to measure the information needed for exponential stabilization for a given set $K$ of initial states: One can look at sets of control functions on $[0, \infty)$ which admit exponential stabilization and at the required bits to discern them on any finite interval $[0, T]$ and then analyze the bit rate for $T \rightarrow \infty$; finally, one can minimize the bit rate over such sets of controls. Alternatively, one may look at sets of control functions defined on a bounded interval $[0, T]$ admitting exponential decay on $[0, T]$ and then look at the minimal number of bits for such a set of control functions. Then, again, one can analyze what happens for $T \rightarrow \infty$. In the first case we speak about minimal bit rates, and in the second case, we speak about entropy, since this approach is close to entropy in the theory of dynamical systems.

\footnotetext{
* Received by the editors April 1, 2011; accepted for publication (in revised form) August 3, 2012; published electronically October 2, 2012.

http://www.siam.org/journals/sicon/50-5/82927.html

†Institut für Mathematik, Universität Augsburg, 86135 Augsburg, Germany (fritz.colonius@math. uni-ausburg.de). This author was supported by DFG grant Co 124/17-2 within DFG Priority Program 1305, Control Theory of Digitally Networked Dynamical Systems.
}

2988 
A discouraging example (Example 2.1) and a general result for linear control systems show that finitely many controls are not sufficient for an exponential estimate on a finite interval. Hence we impose somewhat weaker conditions. Then the stabilization entropy can be shown to be finite; see Theorem 3.3. In the linear case, a formula in terms of eigenvalues holds. It is worth emphasizing that this formula pertains to a fixed exponential decay rate; see Theorem 4.2. Thus it also applies to cases where the control goal is to increase the exponential decay rate for a stable system, a situation where finite communication channels might more easily be tolerated. On the other hand, the stabilization entropy provides an upper bound for the minimal bit rate. In particular, the minimal bit rate is finite (see Theorem 5.3), and in the linear case, an explicit estimate is available.

In section 2 stabilization entropy and minimal bit rates for stabilization are defined and the approach taken in the present paper is explained in more detail. Section 3 provides upper and lower bounds for the stabilization entropy. Section 4 characterizes the stabilization entropy for linear control systems using the sum of the real parts of the eigenvalues exceeding the exponential decay rate. Finally, section 5 shows that the minimal bit rates are equal to or less than the stabilization entropy. For linear systems, conditions are indicated which ensure that both values coincide.

Notation. The closure of a set $A$ is $\operatorname{cl} A$, and the cardinality of a set $A$ is \#A; thus if $A$ is a finite set, $\# A$ is the number of its elements and otherwise $\# A=\infty$. The limit superior and the limit inferior are denoted by $\varlimsup$ lim and $\underline{l}$, respectively.

2. Minimal bit rates and entropy for stabilization. This section discusses the problem formulation and introduces the concepts of minimal bit rates and entropy for exponential stabilization of a control system about an equilibrium. In particular, Proposition 2.2 and Lemma 2.3 motivate the technical formulation.

Consider a nonlinear control system of the form

$$
\dot{x}(t)=f(x(t), u(t)), u \in \mathcal{U},
$$

where $f: \mathbb{R}^{d} \times \mathbb{R}^{m} \rightarrow \mathbb{R}^{d}$ is continuous and Lipschitz continuous with respect to the first argument; the admissible controls are given by

$$
\mathcal{U}=\left\{u:[0, \infty) \rightarrow \mathbb{R}^{m} \mid u(t) \in U \text { for almost all } t \geq 0\right\},
$$

where we assume that the controls $u$ are integrable on every bounded interval and the control range $U$ is a subset of $\mathbb{R}^{m}$. We assume that (i) unique global solutions $\varphi\left(t, x_{0}, u\right), t \geq 0$, of the differential equation with initial condition $x(0)=x_{0} \in \mathbb{R}^{d}$ and control $u \in \mathcal{U}$ exist and (ii) on compact intervals, the solutions depend continuously on the initial value.

We consider the bit rates for the problem to stabilize an equilibrium $x^{*} \in \mathbb{R}^{d}$ corresponding to an admissible control value $u^{*} \in U$, i.e., $0=f\left(x^{*}, u^{*}\right)$. Without loss of generality the equilibrium is given by $x^{*}=0$ corresponding to the control value $u^{*}=0 \in U$. Suppose that the system is exponentially controllable to the equilibrium for all $x_{0}$ in a neighborhood of $x^{*}=0$, i.e., there are constants $M, \alpha>0$ such that for all $x_{0} \neq 0$ there is $u \in \mathcal{U}$ with

$$
\left\|\varphi\left(t, x_{0}, u\right)\right\|<M e^{-\alpha t}\left\|x_{0}\right\| \text { for all } t \geq 0 .
$$

This holds, for example, if the linearized system is stabilizable by a linear feedback; see, e.g., Sontag [19, section 5.8] for asymptotic stability and Grüne and Junge 
[9, Satz 9.8] for exponential stability. For linear control systems, an estimate of the form (2.2) holds iff the unstable part is controllable.

We are interested in problems where the controller does not have continual access to the present state $x(t)$ (or to observed values $y(t)=g(x(t)$ ).) Instead, suppose that the communication channel between the system and the controller only admits a finite bit rate. Then it is not justified that the controller generates infinitely many different control functions on a finite time interval, since only a finite amount of information is available. This excludes state dependent controls $u\left(x\left(t, x_{0}\right)\right)$, where depending on the initial states $x_{0}$ one obtains infinitely many control functions. Instead, the controller might use information on the system to compute open loop controls on some time interval $\left[t_{i}, t_{i+1}\right]$; then, using updated information, a new control function might be computed and used on the next time interval $\left[t_{i+1}, t_{i+2}\right]$. (The $t_{i}$ might depend on the initial value $x_{0}$.) The computations may be based on quantization of the state space or symbolic controllers or may be done via devices like model predictive control (MPC). In any case, this results in time dependent control functions $u$ defined on $[0, \infty)$. For every initial value, one of these control functions should yield the desired decay rate. Instead of concentrating on the algorithmic question of how to generate these controls, we discuss the minimal bit rate needed to discern the time dependent control functions $u$ on any time interval $[0, T], T>0$. This, in fact, is the point of view taken in Tatikonda and Mitter [20, p. 1057], who estimate the minimal bit rate for stabilization of discrete time linear systems from below; see [20, Proposition 3.2].

The following example (the simplest controllable system) shows that a direct approach is not possible. Here on a finite time interval $[0, T]$ one cannot find finitely many controls such that for every point in a neighborhood of the origin exponential estimate (2.2) is satisfied.

Example 2.1. Consider the following scalar system:

$$
\dot{x}=u, u \in U=\mathbb{R}
$$

Let $\alpha>0, M>1$ and fix $T>0$. We claim that there is no finite set $\mathcal{S}$ of control functions on $[0, T]$, such that for every $0 \neq x_{0} \in K:=[-1,1]$ there is $u \in \mathcal{S}$ such that the exponential estimate

$$
\left|\varphi\left(t, x_{0}, u\right)\right|=\left|x_{0}+\int_{0}^{t} u(s) d s\right|<M e^{-\alpha t}\left|x_{0}\right| \text { for all } t \in[0, T]
$$

holds. We proceed by contradiction: Suppose that a finite set $\mathcal{S}=\left\{u_{1}, \ldots, u_{n}\right\}$ with the stated properties exists and define

$$
K_{j}:=\left\{x_{0} \in K \mid\left\|\varphi\left(t, x_{0}, u_{j}\right)\right\|<M e^{-\alpha t}\left\|x_{0}\right\| \text { for all } t \in[0, T]\right\} .
$$

Observe that $0 \notin K_{j}$. For the control $u_{0}(t) \equiv 0$, every point $x_{0}$ is an equilibrium. Thus for $x_{0} \neq 0$ the control $u_{0}$ does not satisfy (2.4) if $T$ is large enough such that $M e^{-\alpha T}<1$, and we may assume that $u_{0} \notin \mathcal{S}$. Hence for every $j$ one finds $t_{j} \in[0, T]$ with

$$
c_{j}:=\max _{t \in[0, T]}\left|\int_{0}^{t} u_{j}(s) d s\right|=\left|\int_{0}^{t_{j}} u_{j}(s) d s\right|>0 .
$$

Consider an initial point $x_{0} \in K$ with $\left|x_{0}\right|<\min _{j} \frac{c_{j}}{2 M}$. Observe that then $\left|x_{0}\right|<$ $M\left|x_{0}\right|<\frac{1}{2} \min _{j} c_{j}$, since $M>1$. We claim that there is no control $u_{j} \in \mathcal{S}$ satisfying 
(2.4). In fact, one computes

$$
\left|x_{0}+\int_{0}^{t_{j}} u_{j}(s) d s\right| \geq\left|\int_{0}^{t_{j}} u_{j}(s) d s\right|-\left|x_{0}\right| \geq c_{j}-\frac{c_{j}}{2}=\frac{c_{j}}{2}>e^{-\alpha t_{j}} \frac{c_{j}}{2}>M e^{-\alpha t_{j}}\left|x_{0}\right| .
$$

An easy generalization of this example shows that the same result valid for any linear control system.

Proposition 2.2. Consider a linear control system of the form

$$
\dot{x}=A x+B u
$$

with $A \in \mathbb{R}^{d \times d}, B \in \mathbb{R}^{d \times m}$ and controls $u:[0, \infty) \rightarrow \mathbb{R}^{m}$ which are integrable on every bounded interval. Assume that there is an eigenvalue $\lambda$ of $A$ with $\operatorname{Re} \lambda \geq 0$. Let $\alpha>0, M>1$, consider a neighborhood $K$ of the origin, and fix $T>0$. Then there is no finite set $\mathcal{S}$ of control functions such that for every $0 \neq x_{0} \in K$ there is $u \in \mathcal{S}$ with

$$
\left\|\varphi\left(t, x_{0}, u\right)\right\|<M e^{-\alpha t}\left\|x_{0}\right\| \text { for all } t \in[0, T] .
$$

Proof. Again we proceed by contradiction. Suppose that a finite set $\mathcal{S}=$ $\left\{u_{1}, \ldots, u_{n}\right\}$ of control functions with the stated property exists and define $K_{j}$ as in (2.5). Consider $x_{0} \neq 0$ in the real eigenspace $E(\lambda)$ for $\lambda$. Then, for $T$ large enough such that $M e^{-\alpha T}<1$, any control $u_{0}$ with $\int_{0}^{t} e^{A(t-s)} B u_{0}(s) d s \equiv 0$ will not yield estimate (2.6). Hence, we may assume that the controls $u_{j} \in \mathcal{S}, j \in J \subset\{1, \ldots, n\}$ which for any $0 \neq x_{0} \in K \cap E(\lambda)$ yield estimate (2.6) satisfy for some $t_{j} \in[0, T]$

$$
c_{j}:=\max _{t \in[0, T]}\left\|\int_{0}^{t} e^{A(t-s)} B u_{j}(s) d s\right\|=\left\|\int_{0}^{t_{j}} e^{A\left(t_{j}-s\right)} B u_{j}(s) d s\right\|>0 .
$$

Choose $0 \neq x_{0} \in K \cap E(\lambda)$ with $\left\|x_{0}\right\|<\min _{j \in J} \frac{c_{j}}{2 M}$. We find for every $j \in J$ the contradiction

$$
\begin{aligned}
\left\|\varphi\left(t_{j}, x_{0}, u_{j}\right)\right\| & =\left\|x_{0}+\int_{0}^{t_{j}} e^{A\left(t_{j}-s\right)} B u_{j}(s) d s\right\| \geq\left\|\int_{0}^{t_{j}} e^{A\left(t_{j}-s\right)} B u_{j}(s) d s\right\|-\left\|x_{0}\right\| \\
& \geq c_{j}-\frac{c_{j}}{2}=\frac{c_{j}}{2} \geq e^{-\alpha t_{j}} \frac{c_{j}}{2}>M e^{-\alpha t_{j}}\left\|x_{0}\right\|
\end{aligned}
$$

In contrast to the linear example (2.3), the scalar bilinear system

$$
\dot{x}=(1+u) x, u \in U=\mathbb{R},
$$

can be stabilized by the single constant control $u(t) \equiv-2$. Thus a single bit is sufficient. See also de Persis [6] for other situations where finitely many bits are sufficient. While it might be worthwhile to study bilinear control systems in this context, we follow a different path in the rest of this paper and relax the exponential stability property (2.2) by introducing a small additive term. The following simple but basic lemma shows that then only finitely many bits are required on a finite interval.

Lemma 2.3. Consider a control system of the form (2.1) and let $K$ be a compact subset of $\mathbb{R}^{d}$. Assume that there are constants $M>1$ and $\alpha>0$ such that for all $0 \neq x \in K$ there is $u \in \mathcal{U}$ with

$$
\|\varphi(t, x, u)\|<M e^{-\alpha t}\|x\| \text { for all } t \geq 0 .
$$

Copyright $@$ by SIAM. Unauthorized reproduction of this article is prohibited. 
Let $\varepsilon>0$. Then for every $T>0$ there is a finite set $\mathcal{S}=\left\{u_{1}, \ldots, u_{n}\right\} \subset \mathcal{U}$ such that for every $x \in K$ there is $u_{j} \in \mathcal{S}$ with

$$
\left\|\varphi\left(t, x, u_{j}\right)\right\|<e^{-\alpha t}(\varepsilon+M\|x\|) .
$$

Proof. For every $x_{0} \in K$ choose a control $u \in \mathcal{U}$ with

$$
\left\|\varphi\left(t, x_{0}, u\right)\right\|<M e^{-\alpha t}\left\|x_{0}\right\| \text { for all } t \in[0, T] .
$$

By continuous dependence on initial values (as assumed for (2.1)) there is $\delta$ with $0<\delta<\varepsilon / M$ such that for all $x \in \mathbb{R}^{d}$ with $\left\|x_{0}-x\right\|<\delta$ one has for all $t \in[0, T]$

$$
\begin{aligned}
\|\varphi(t, x, u)\| & <M e^{-\alpha t}\left\|x_{0}\right\| \leq M e^{-\alpha t}\left(\left\|x_{0}-x\right\|+\|x\|\right) \\
& <M e^{-\alpha t}(\delta+\|x\|) \\
& <e^{-\alpha t}(\varepsilon+M\|x\|) .
\end{aligned}
$$

Now compactness of $K$ shows that there is a finite set $\mathcal{S}=\left\{u_{1}, \ldots, u_{n}\right\} \subset \mathcal{U}$ such that for each $x \in K$ there is $u_{j} \in \mathcal{S}$ satisfying for all $t \in[0, T]$

$$
\left\|\varphi\left(t, x, u_{j}\right)\right\|<e^{-\alpha t}(\varepsilon+M\|x\|) .
$$

In view of the preceding lemma, we will consider weakened versions of estimate (2.7). There are the two ways to measure the information needed for stabilization, and we begin with an entropy-like notion. Consider a compact set $K \subset \mathbb{R}^{d}$ of initial states, and let $\alpha>0, M>1$ and $\varepsilon>0$. For a time $T>0$ we call a subset $\mathcal{S} \subset \mathcal{U}$ a $(T, \varepsilon, \alpha, M, K)$-spanning set of controls if for all $x \in K$ there is $u \in \mathcal{S}$ with

$$
\|\varphi(t, x, u)\|<e^{-\alpha t}(\varepsilon+M\|x\|) \text { for all } t \in[0, T] .
$$

We denote the cardinality of $\mathcal{S}$ by the symbol $\# \mathcal{S}$, and the minimal cardinality of all these sets is

$$
s_{\text {stab }}(T, \varepsilon, \alpha, M, K):=\min \{\# \mathcal{S} \mid \mathcal{S} \text { is }(T, \varepsilon, \alpha, M, K) \text {-spanning }\} .
$$

If there is no finite set $\mathcal{S}$ of controls with this property (in particular, if there is no such set at all), we let $s_{\text {stab }}(T, \varepsilon, \alpha, M, K):=\infty$. Note that for $\varepsilon_{1}>\varepsilon_{2}>$ 0 , any $\left(T, \varepsilon_{2}, \alpha, M, K\right)$-spanning set is also $\left(T, \varepsilon_{1}, \alpha, M, K\right)$-spanning. Lemma 2.3 shows that exponential controllability condition (2.7) implies the existence of finite $(T, \varepsilon, \alpha, M, K)$-spanning sets. We want to determine which information has to be transmitted through a digital communication channel in order to identify a control function in such a finite set $\mathcal{S}$. The elements can be encoded by symbols given by finite sequences of 0 's and 1's in the set

$$
\Sigma_{k}:=\left\{\left(s_{0} s_{1} s_{2} \ldots s_{k-1}\right) \mid s_{i} \in\{0,1\} \text { for } i=0,1, \ldots, k-1\right\},
$$

where $k \in \mathbb{N}$ is the least integer greater than or equal to $\log _{2} \# \mathcal{S}$. Thus $\# \mathcal{S}$ is bounded above by $2^{k}$. Equivalently, the number of bits determining an element of $\mathcal{S}$ is $\log _{2}\left(2^{k}\right)=k$. It will be convenient to use here the natural logarithm instead of the logarithm with base 2. It allows us to avoid the annoying factor $\log _{2} e$ in section 4 . (Regrettably, it will show up in section 5.) Now we consider what happens for time tending to infinity and then $\varepsilon$ tending to 0 to obtain the following notion describing an entropy property for exponential stabilization. 
Definition 2.4. Let $K$ be a compact set in $\mathbb{R}^{d}$ and $\alpha, M>0$. Then the stabilization entropy $h_{\mathrm{stab}}(\alpha, M, K)$ is defined by

$$
h_{\text {stab }}(\alpha, M, K)=\lim _{\varepsilon \searrow 0} \varlimsup_{T \rightarrow \infty} \frac{1}{T} \ln s_{\text {stab }}(T, \varepsilon, \alpha, M, K) .
$$

In the following, we drop the argument $K$ in this and in similar notions if the choice of $K$ is clear or if its specification is irrelevant in the corresponding context. The existence of the limit for $\varepsilon \searrow 0$ is obvious, since it equals the supremum over all $\varepsilon>0$. (The value $+\infty$ is allowed.) Furthermore, the inequality $h_{\text {stab }}\left(\alpha^{\prime}, M^{\prime}\right) \leq h_{\text {stab }}(\alpha, M)$ holds for $\alpha \geq \alpha^{\prime}>0$ and $M^{\prime} \geq M>0$.

Remark 2.5. If one would consider $\alpha=0$, condition (2.9) just means that every trajectory starting in $K$ remains in the ball around the origin with radius $\varepsilon+$ $M \max _{x \in K}\|x\|$. In this case, the results on invariance entropy from Kawan $[11,12,13]$ would apply.

A second way of counting bits is the following. Consider a set of control functions defined on $[0, \infty)$ which allow us to steer the system asymptotically to the equilibrium $x^{*}=0$ satisfying the following conditions. Let $M>1, \alpha>0, \varepsilon>0$ and let $\gamma$ be a decreasing function on $[0, \infty)$ with $\gamma(0)=\varepsilon$ and $\lim _{t \rightarrow \infty} \gamma(t)=0$. For brevity, we call $\gamma$ an $\mathcal{L}_{\varepsilon}$-function. (Note that continuity of $\gamma$ is not required.) Let $\mathcal{R}=\mathcal{R}(\gamma, \varepsilon, \alpha, M) \subset$ $\mathcal{U}$ be a set of of control functions such that for all $x \in K$ there is $u \in \mathcal{R}$ with

$$
\|\varphi(t, x, u)\|<\gamma(t)+M e^{-\alpha t}\|x\| \text { for all } t \geq 0 .
$$

Then $\mathcal{R}$ is called $(\gamma, \varepsilon, \alpha, M)$-stabilizing for $K$. Thus in the $\varepsilon$ - neighborhood of the equilibrium, the decay given by the exponential rate $\alpha$ may slow down, but still convergence holds for $t \rightarrow \infty$. Let

$$
\mathcal{R}_{T}:=\left\{u_{\mid[0, T]} \mid u \in \mathcal{R}\right\}
$$

be the corresponding restrictions of the controls in $\mathcal{R}$. Suppose that the cardinality $\# \mathcal{R}_{T}$ is finite. (Lemma 5.2 will provide conditions when this holds.) The bit rate on the time interval $[0, T]$ is defined as $\frac{1}{T} \log _{2} \# \mathcal{R}_{T}$. If there is no set $\mathcal{R}$ with $(2.11)$ or if $\mathcal{R}_{T}$ contains infinitely many elements, we set $\# \mathcal{R}_{T}:=\infty$. The required bit rate for stabilization using controls in $\mathcal{R}=\mathcal{R}(\gamma, \varepsilon, \alpha, M)$ is

$$
b(\mathcal{R}(\gamma, \varepsilon, \alpha, M)):=\underline{\lim }_{T \rightarrow \infty} \frac{1}{T} \log _{2} \# \mathcal{R}_{T}(\gamma, \varepsilon, \alpha, M) .
$$

DEFINITION 2.6. With the notions introduced above, the minimal bit rate for $(\alpha, M)$-stabilization at $x^{*}=0$ of a compact set $K \subset \mathbb{R}^{d}$ is

$$
b_{\text {stab }}(\alpha, M):=\lim _{\varepsilon \searrow 0} \inf _{\gamma \in \mathcal{L}_{\varepsilon}} \inf _{\mathcal{R}(\gamma, \varepsilon, \alpha, M)} b(\mathcal{R}(\gamma, \varepsilon, \alpha, M)),
$$

where the inner infimum is taken over all $(\gamma, \varepsilon, \alpha, M)$-stabilizing sets $\mathcal{R}(\gamma, \varepsilon, \alpha, M) \subset$ $\mathcal{U}$ of controls and the outer infimum is taken over all $\mathcal{L}_{\varepsilon}$-functions $\gamma$.

The limit for $\varepsilon \searrow 0$ is the supremum for $\varepsilon>0$. Note also the inequality $b_{\text {stab }}\left(\alpha^{\prime}, M^{\prime}\right) \leq b_{\text {stab }}(\alpha, M)$ for $\alpha \geq \alpha^{\prime}>0$ and $M^{\prime} \geq M>0$.

An example for an allowed $\mathcal{L}_{\varepsilon}$-functions is $\gamma(t)=\varepsilon e^{-\alpha t}, t \geq 0$. However, for this function, we cannot prove our main result for the stabilization bit rate (Theorem 5.3) showing that the stabilization entropy provides an upper bound. Also, this theorem will only give a result for the limit inferior for $T \rightarrow \infty$ (not for the limit superior). Consideration of the limit inferior may be justified by the fact that we are interested in the minimal bit rate for $T \rightarrow \infty$, hence the times may be chosen appropriately. 
The stabilization entropy indicates how much the number of required control functions increases when time increases. Here minimization is performed on each interval $[0, T]$ separately. If one wants to enlarge the time interval where the exponential decay holds, one may have to consider controls which, when restricted to the smaller interval, are different from the earlier ones. This is in contrast to minimal bit rates, where restrictions to $[0, T]$ are considered for control functions defined on $[0, \infty)$. Thus, while stabilization entropy certainly merits its own interest, the minimal bit rate might appear more appealing from this point of view.

Remark 2.7. The difference between these two concepts can also be seen by looking at them from a quantization point of view. Let $\mathcal{R}$ be a $(\gamma, \varepsilon, \alpha, M)$-stabilizing set such that for every $T>0$ the set $\mathcal{R}_{T}$ of restrictions to $[0, T]$ is finite. Then define for every $u \in \mathcal{R}_{T}$

$$
K(u, T):=\left\{x \in K \mid\|\varphi(t, x, u)\|<\gamma(t)+M e^{-\alpha t}\|x\| \text { for all } t \in[0, T]\right\} .
$$

The sets $K(u, T)$ form an open cover of $K$ which may be viewed as a finite quantization. For $T^{\prime}>T$, the same construction for $\mathcal{R}_{T^{\prime}}$ again yields a finite quantization of $K$ which is obtained by refining the quantization at time $T$, since both are obtained by restrictions of controls in $\mathcal{R}$. In contrast, the quantization for $T^{\prime}>T$ obtained by a $\left(T^{\prime}, \varepsilon, \alpha, M\right)$-spanning set of controls used for defining the entropy is not related to the quantization associated with a $(T, \varepsilon, \alpha, M)$-spanning set.

Remark 2.8. With $\log _{2} e \cdot \ln x=\log _{2} x$ one immediately finds

$$
b_{\text {stab }}(\alpha, M)=\log _{2} e \cdot \lim _{\varepsilon \searrow 0} \inf _{\gamma \in \mathcal{L}_{\varepsilon}} \inf _{\mathcal{R}(\gamma, \varepsilon, \alpha, M)} \underline{\lim }_{T \rightarrow \infty} \frac{1}{T} \ln \# \mathcal{R}_{T}(\gamma, \varepsilon, \alpha, M) .
$$

In the next two sections we will first concentrate on the stabilization entropy, which is easier to analyze than the minimal bit rate.

3. Upper and lower estimates. In this section, lower estimates for the stabilization entropy and the minimal bit rate are proven. For the entropy, also an upper bound can be established, which, in particular, shows that it is finite.

The following proposition explains the behavior of stabilization entropy when the set of initial values is changed, and it shows that it suffices to consider multiples of a fixed time step. These properties which are analogous to properties of invariance entropy (cf. Colonius and Kawan [2, Proposition 3.4(ii) and (iii)]) are not used below.

Proposition 3.1. Consider a system of the form (2.1), let $K \subset \mathbb{R}^{d}$ be a compact set, and fix $\alpha>0$ and $M>1$.

(i) Then for all $\varepsilon>0$ and $\tau>0$

$$
\varlimsup_{T \rightarrow \infty} \frac{1}{T} \ln s_{\text {stab }}(T, \varepsilon, \alpha, M)=\varlimsup_{N \rightarrow \infty} \frac{1}{N \tau} \ln s_{\text {stab }}(N \tau, \varepsilon, \alpha, M) .
$$

(ii) Let $K_{i} \subset K, i=1, \ldots, N$, be closed subsets of $K$ with $K=\bigcup_{i=1}^{N} K_{i}$. Then the stabilization entropies with respect to $K$ and $K_{i}$, respectively, satisfy

$$
h_{\mathrm{stab}}(\alpha, M, K)=\max _{i=1, \ldots, N} h_{\mathrm{stab}}\left(\alpha, M, K_{i}\right)
$$

Proof. (i) Obviously, the left-hand side of (3.1) is not less than the right-hand side. In order to show the reverse inequality, let $\left(T_{k}\right)_{k \in \mathbb{N}}$ be a sequence converging to $\infty$. Then for every $k \in \mathbb{N}$ there exists $n_{k} \in \mathbb{N}$ such that $n_{k} \tau \leq T_{k}<\left(n_{k}+1\right) \tau$, 
and $n_{k} \rightarrow \infty$ for $k \rightarrow \infty$. If $S \leq T$, then $s_{\text {stab }}(S, \varepsilon, \alpha, M) \leq s_{\text {stab }}(T, \varepsilon, \alpha, M)$, which implies

$$
s_{\text {stab }}\left(T_{k}, \varepsilon, \alpha, M\right) \leq s_{\text {stab }}\left(\left(n_{k}+1\right) \tau, \varepsilon, \alpha, M\right)
$$

and consequently

$$
\frac{1}{T_{k}} \ln s_{\text {stab }}\left(T_{k}, \varepsilon, \alpha, M\right) \leq \frac{1}{n_{k} \tau} \ln s_{\text {stab }}\left(\left(n_{k}+1\right) \tau, \varepsilon, \alpha, M\right) .
$$

Since $\frac{1}{n_{k} \tau}=\frac{n_{k}+1}{n_{k}} \frac{1}{\left(n_{k}+1\right) \tau}$ and $\frac{n_{k}+1}{n_{k}} \rightarrow 1$ for $k \rightarrow \infty$, we obtain assertion (i)

$$
\begin{aligned}
\varlimsup_{k \rightarrow \infty} \frac{1}{T_{k}} \ln s_{\mathrm{stab}}\left(T_{k}, \varepsilon, \alpha, M\right) & \leq \varlimsup_{k \rightarrow \infty} \frac{1}{n_{k} \tau} \ln s_{\mathrm{stab}}\left(\left(n_{k}+1\right) \tau, \varepsilon, \alpha, M\right) \\
& =\varlimsup_{N \rightarrow \infty} \frac{1}{N \tau} \ln s_{\mathrm{stab}}(N \tau, \varepsilon, \alpha, M) .
\end{aligned}
$$

(ii) Every minimal $(T, \varepsilon, \alpha, M, K)$-spanning set $\mathcal{S}$ is also $\left(T, \varepsilon, \alpha, M, K_{i}\right)$-spanning. Thus we obtain $s_{\text {stab }}\left(T, \varepsilon, \alpha, M, K_{i}\right) \leq s_{\text {stab }}(T, \varepsilon, \alpha, M, K)$ implying

$$
\max _{i=1, \ldots, N} h_{\mathrm{stab}}\left(\alpha, M, K_{i}\right) \leq h_{\mathrm{stab}}(\alpha, M, K) \text {. }
$$

On the other hand, if $\mathcal{S}_{i}$ is a minimal $\left(T, \varepsilon, \alpha, M, K_{i}\right)$-spanning set, $i=1, \ldots, N$, then $\mathcal{S}:=\bigcup_{i=1}^{N} \mathcal{S}_{i}$ is $(T, \varepsilon, \alpha, M, K)$-spanning. This yields

$$
s_{\text {stab }}(T, \varepsilon, \alpha, M, K) \leq \# \mathcal{S} \leq \sum_{i=1}^{N} \# \mathcal{S}_{i}=\sum_{i=1}^{N} s_{\text {stab }}\left(T, \varepsilon, \alpha, M, K_{i}\right) .
$$

By a standard property of such limits (see, e.g., [2, Lemma 3.3]) we obtain

$$
\varlimsup_{T \rightarrow \infty} \frac{1}{T} \ln \sum_{i=1}^{N} s_{\text {stab }}\left(T, \varepsilon, \alpha, M, K_{i}\right)=\max _{i=1, \ldots, N} \varlimsup_{T \rightarrow \infty} \frac{1}{T} \ln s_{\text {stab }}\left(T, \varepsilon, \alpha, M, K_{i}\right)
$$

implying the desired result

$$
\begin{aligned}
h_{\text {stab }}(\alpha, M, K) & \leq \lim _{\varepsilon \searrow 0} \varlimsup_{T \rightarrow \infty} \frac{1}{T} \ln \sum_{i=1}^{N} s_{\text {stab }}\left(T, \varepsilon, \alpha, M, K_{i}\right) \\
& \leq \max _{i=1, \ldots, N} h_{\text {stab }}\left(\alpha, M, K_{i}\right) .
\end{aligned}
$$

Next we will provide lower and upper bounds for the $(\alpha, M)$-stabilization entropy $h_{\text {stab }}(\alpha, M)$. In the following theorem, we denote the divergence of a function $f$ with respect to the first variable by

$$
\operatorname{div}_{x} f(x, u)=\sum_{i=1}^{d} \frac{\partial f_{i}}{\partial x_{i}}(x, u)=\operatorname{tr} f_{x}(x, u),
$$

where $f_{1}, \ldots, f_{d}: \mathbb{R}^{d} \times \mathbb{R}^{m} \rightarrow \mathbb{R}$ are the coordinate functions of $f$ assumed to be differentiable with respect to $x$.

Let $\beta \in \mathbb{R}_{+}=[0, \infty)$ be given and introduce a set $\mathcal{V}=\mathcal{V}(\beta)$ of admissible controls which has the form

$$
\mathcal{V}:=\left\{v:[0, \infty) \rightarrow \mathbb{R}^{m} \mid \text { there is } u \in \mathcal{U} \text { with } v(t)=e^{\beta t} u(t) \text { for all } t \geq 0\right\} .
$$

Copyright ( by SIAM. Unauthorized reproduction of this article is prohibited. 
This generalized set of control functions will be needed in section 4 . Note that for $\beta=0$, the set $\mathcal{V}$ reduces to $\mathcal{U}$. Naturally, $\mathcal{V}=\mathcal{U}$ also holds for unconstrained control range $U=\mathbb{R}^{m}$. The definition of stabilization entropy can, with only trivial changes, be extended to control systems with admissible controls in $\mathcal{V}$.

THEOREM 3.2. Consider control system (2.1) with controls $v$ in a set $\mathcal{V}$ of the form (3.2) and let $x^{*}=0$ be an equilibrium with $0=f(0,0), 0 \in U$. Suppose that $f$ is $C^{1}$ with respect to $x$ and that the derivative $f_{x}(x, u)$ is continuous in $(x, u)$. Let $K \subset \mathbb{R}^{d}$ be a compact set with positive Lebesgue measure $\lambda(K)$ and denote $\kappa:=$ $\max _{x \in K}\|x\|$. Let $\alpha, M>0$ and assume that $\operatorname{div}_{x} f(x, v)$ is bounded below for $\|x\| \leq$ $M \kappa+1$ and $v \in V:=\{a u \mid a \geq 0$ and $u \in U\}$. Then the stabilization entropy and the minimal bit rate for stabilization of $K$ satisfies the estimates

$$
h_{\mathrm{stab}}(\alpha, M) \geq \alpha d+\min _{v \in V} \operatorname{div}_{x} f(0, v) \text { and } b_{\mathrm{stab}}(\alpha, M) \geq \log _{2} e \cdot \min _{v \in V} \operatorname{div}_{x} f(0, v) .
$$

Proof. If $h_{\text {stab }}(\alpha, M)=\infty$, inequality (3.3) is trivially satisfied. Hence we may assume that for $\varepsilon>0, M>0$ and $T>0$ there is a finite $(T, \varepsilon, \alpha, M)$-spanning set $\mathcal{S}=$ $\left\{v_{1}, \ldots, v_{s}\right\} \subset \mathcal{V}$, and we pick $\mathcal{S}$ with minimal cardinality, hence $s=s_{\text {stab }}(T, \varepsilon, \alpha, M)$. Define for $j=1, \ldots, s$ the set $K_{j}$ as the set of all $x \in K$ with

$$
\left\|\varphi\left(t, x, v_{j}\right)\right\|<\delta(t):=e^{-\alpha t}(\varepsilon+M\|x\|) \text { for all } t \in[0, T] .
$$

One sees that with $\delta(t):=e^{-\alpha t}(\varepsilon+M \kappa)$ for $j=1, \ldots, s$

$$
\varphi\left(t, K_{j}, v_{j}\right) \subset\left\{y \in \mathbb{R}^{d} \mid\|y\|<\delta(t)\right\}=: \mathbf{B}(0 ; \delta(t)) .
$$

In particular, if we consider the ball in the maximum-norm in $\mathbb{R}^{d}$, we obtain for the Lebesgue measures

$$
\lambda\left(\varphi\left(T, K_{j}, v_{j}\right)\right) \leq \lambda(\mathbf{B}(0 ; \delta(T)))=2^{d} \delta(T)^{d} .
$$

On the other hand, by the transformation theorem and Liouville's trace formula we get for all $j \in\{1, \ldots, s\}$

$$
\begin{aligned}
\lambda\left(\varphi\left(T, K_{j}, v_{j}\right)\right) & =\int_{K_{j}}\left|\operatorname{det} \frac{\partial \varphi}{\partial x}\left(T, x, v_{j}\right)\right| d x \\
& \geq \lambda\left(K_{j}\right) \cdot \inf _{(x, v)}\left|\operatorname{det} \frac{\partial \varphi}{\partial x}(T, x, v)\right| \\
& =\lambda\left(K_{j}\right) \cdot \inf _{(x, v)} \exp \left(\int_{0}^{T} \operatorname{di} v_{x} f(\varphi(\tau, x, v), v(\tau)) d \tau\right) .
\end{aligned}
$$

Here and in the rest of this proof, $\inf _{(x, v)}$ denotes the infimum over all $(x, v) \in K \times \mathcal{V}$ with $\varphi(t, x, v) \subset \operatorname{clB}(0 ; \delta(t))$ for all $t \in[0, T]$. Fix $T_{0} \in[0, T]$. Since $\delta(t) \leq \delta\left(T_{0}\right)$ for $t \geq T_{0}$ one obtains the estimate

$$
\begin{aligned}
& \inf _{(x, v)} \exp \left(\int_{0}^{T} \operatorname{div}_{x} f(\varphi(\tau, x, v), v(\tau)) d \tau\right) \\
& \quad \geq \exp \left(\inf _{(x, v)} \int_{0}^{T_{0}} \operatorname{div}_{x} f(\varphi(\tau, x, v), v(\tau)) d \tau+\left(T-T_{0}\right) \inf _{(y, w)} \operatorname{div}_{x} f(y, w)\right),
\end{aligned}
$$

where the second infimum is taken over all $(y, w) \in \operatorname{cl} \mathbf{B}\left(0 ; \delta\left(T_{0}\right)\right) \times V$. This term is finite by the assumption on the divergence of $f$. Since $\left\|\varphi\left(\tau, x, v_{j}\right)\right\|<M \kappa+1, \tau \in$ 
$\left[0, T_{0}\right]$, our assumption also guarantees that $\operatorname{div}_{x} f(\varphi(\tau, x, v), v(\tau))$ with $(x, v) \in K \times \mathcal{V}$ is bounded below, hence also the first summand in the exponent is finite.

We may assume that $\lambda\left(K_{1}\right)=\max _{j=1, \ldots, s} \lambda\left(K_{j}\right)$. Inequalities (3.6) and (3.5) imply

$$
\begin{aligned}
0 & <\lambda(K) \leq \sum_{j=1}^{s} \lambda\left(K_{j}\right) \leq s \cdot \lambda\left(K_{1}\right) \leq s \cdot \frac{\lambda\left(\varphi\left(T, K_{1}, v_{1}\right)\right)}{\inf _{(x, v)} \exp \left(\int_{0}^{T} \operatorname{div}_{x} f(\varphi(\tau, x, v), v(\tau)) d \tau\right)} \\
& \leq s \cdot \frac{2^{d} \delta(T)^{d}}{\inf _{(x, v)} \exp \left(\int_{0}^{T} \operatorname{div}_{x} f(\varphi(\tau, x, v), v(\tau)) d \tau\right)},
\end{aligned}
$$

hence

$$
s=s_{\text {stab }}(T, \varepsilon, \alpha, M) \geq \frac{\lambda(K)}{2^{d} \delta(T)} \inf _{(x, v)} \exp \left(\int_{0}^{T} \operatorname{div}_{x} f(\varphi(\tau, x, v), v(\tau)) d \tau\right) .
$$

Using (3.7) and taking the logarithm on both sides one finds

$$
\begin{aligned}
& \ln s_{\text {stab }}(T, \varepsilon, \alpha, M) \\
& \geq \ln \lambda(K)-d \ln [2 \delta(T)] \\
& \quad+\inf _{(x, v)}\left(\int_{0}^{T_{0}} \operatorname{div}_{x} f(\varphi(\tau, x, v), v(\tau)) d \tau\right)+\left(T-T_{0}\right) \min _{(y, w)} \operatorname{div}_{x} f(y, w) .
\end{aligned}
$$

Since

$$
\lim _{T \rightarrow \infty} \frac{1}{T} \ln \delta(T)=\lim _{T \rightarrow \infty} \frac{1}{T} \ln \left[2 e^{-\alpha T}(\varepsilon+M \kappa)\right]=-\alpha+\lim _{T \rightarrow \infty} \frac{1}{T} \ln (2 \varepsilon+2 M \kappa)=-\alpha,
$$

one finds the inequality

$$
\begin{aligned}
\varlimsup_{T \rightarrow \infty} \frac{1}{T} \ln s_{\text {stab }}(T, \varepsilon, \alpha, M) & \geq \alpha d+\varlimsup_{T \rightarrow \infty}\left[\frac{T-T_{0}}{T} \min _{(y, w)} \operatorname{div}_{x} f(y, w)\right] \\
& =\alpha d+\min _{(y, w)} \operatorname{div}_{x} f(y, w) .
\end{aligned}
$$

Recall that the minimum is taken over all $(y, w) \in \operatorname{cl} \mathbf{B}\left(0 ; e^{-\alpha T_{0}}(\varepsilon+M \kappa)\right) \times V$. In the Hausdorff metric the set $\operatorname{clB}\left(0 ; e^{-\alpha T_{0}}(\varepsilon+M \kappa)\right)$ converges for $T_{0} \rightarrow \infty$ to $\{0\}$. This together with (3.10) shows inequality (3.3).

For the minimal bit rate, take a $(\gamma, \varepsilon, \alpha, M)$-stabilizing set such that the set $\mathcal{R}_{T}(\gamma, \varepsilon, \alpha, M)=\left\{v_{1}, \ldots, v_{r}\right\}$ of restrictions to $[0, T]$ is finite. Define for $j=1, \ldots, r$ the set $K_{j}$ as the set of all $x \in K$ with

$$
\left\|\varphi\left(t, x, v_{j}\right)\right\|<\gamma(t)+M e^{-\alpha t}\|x\| \text { for all } t \in[0, T] .
$$

Then the proof above can be repeated with $\delta(t)$ in (3.4) replaced by

$$
\delta(t):=\gamma(t)+M e^{-\alpha t} \kappa, t \in[0, T] .
$$

Instead of (3.9), we estimate for $T>0$ large enough

$$
\begin{aligned}
\ln \delta(T) & =\ln \left[\gamma(T)+M e^{-\alpha T} \kappa\right] \leq \ln \left[\varepsilon+M e^{-\alpha T} \kappa\right]=-\alpha T+\ln \left[\varepsilon e^{\alpha T}+M \kappa\right] \\
& \leq-\alpha T+\ln \left[2 \varepsilon e^{\alpha T}\right]=-\alpha T+\alpha T+\ln (2 \varepsilon)=\ln (2 \varepsilon) .
\end{aligned}
$$

Copyright $(\odot$ by SIAM. Unauthorized reproduction of this article is prohibited. 
Hence

$$
\lim _{T \rightarrow \infty} \frac{1}{T} \ln \delta(T) \leq 0
$$

and the analogue of (3.8) shows that

$$
\varlimsup_{T \rightarrow \infty} \frac{1}{T} \ln \# \mathcal{R}_{T}(\gamma, \varepsilon, \alpha, M) \geq \varlimsup_{T \rightarrow \infty}\left[\frac{T-T_{0}}{T} \min _{(y, w)} \operatorname{div}_{x} f(y, w)\right]=\min _{(y, w)} \operatorname{div}_{x} f(y, w) .
$$

Then one concludes as above.

The next theorem, whose proof is a modification of Katok and Hasselblatt [10, Theorem 3.3.9] and Colonius and Kawan [2, Theorem 4.2], shows in particular that the stabilization entropy is finite.

Theorem 3.3. Consider a control system of the form (2.1) and let $x^{*}=0$ be an equilibrium with $0=f(0,0), 0 \in U \subset \mathbb{R}^{m}$ and fix $\alpha>0$ and $M>1$. Assume that $K \subset \mathbb{R}^{d}$ is a compact neighborhood of the origin such that for every $0 \neq x \in K$ there is a control $u \in \mathcal{U}$ with

$$
\|\varphi(t, x, u)\|<M e^{-\alpha t}\|x\| \text { for all } t \geq 0 .
$$

Furthermore, suppose that there is a Lipschitz constant $L>0$ such that for all $x_{1}, x_{2}$ in an open set containing $K$

$$
\left\|f\left(x_{1}, u\right)-f\left(x_{2}, u\right)\right\| \leq L\left\|x_{1}-x_{2}\right\| \text { for all } u \in U .
$$

Then the stabilization entropy of $K$ satisfies

$$
h_{\mathrm{stab}}(\alpha, M) \leq(L+\alpha) d .
$$

Proof. Let $\varepsilon>0$ and consider an open cover $\mathcal{C}$ of $K$ by balls $\mathbf{B}\left(x_{i} ; e^{-(L+\alpha) T} \varepsilon / M\right)$ centered at points $x_{i} \in K$ with $i=1, \ldots, N:=\# \mathcal{C}$. Without loss of generality, we may assume that all $x_{i} \neq 0$. By our hypothesis, we can assign to the point $x_{i}$ a control function $u_{i} \in \mathcal{U}$ with

$$
\left\|\varphi\left(t, x_{i}, u_{i}\right)\right\|<M e^{-\alpha t}\left\|x_{i}\right\| \text { for all } t \in[0, T] .
$$

Then for all $x \in \mathbf{B}\left(x_{i} ; e^{-(L+\alpha) T} \varepsilon / M\right)$ and all $t \in[0, T]$

$$
\left\|\varphi\left(t, x, u_{i}\right)-\varphi\left(t, x_{i}, u_{i}\right)\right\| \leq\left\|x-x_{i}\right\|+L \int_{0}^{t}\left\|\varphi\left(\tau, x, u_{i}\right)-\varphi\left(\tau, x_{i}, u_{i}\right)\right\| d \tau
$$

implying by Gronwall's inequality that for all $t \in[0, T]$

$$
\left\|\varphi\left(t, x, u_{i}\right)-\varphi\left(t, x_{i}, u_{i}\right)\right\| \leq\left\|x-x_{i}\right\| e^{L t}<e^{-(L+\alpha) T} \varepsilon e^{L T} \leq e^{-\alpha t} \varepsilon .
$$

It follows that for every $x \in K$ there is $u_{i}$ such that for all $t \in[0, T]$

$$
\begin{aligned}
\left\|\varphi\left(t, x, u_{i}\right)\right\| & \leq\left\|\varphi\left(t, x, u_{i}\right)-\varphi\left(t, x_{i}, u_{i}\right)\right\|+\left\|\varphi\left(t, x_{i}, u_{i}\right)\right\| \\
& \leq e^{-\alpha t} \varepsilon+M e^{-\alpha t}\left\|x_{i}\right\| \\
& \leq e^{-\alpha t}\left(\varepsilon+M\left\|x_{i}-x\right\|+M\|x\|\right) \\
& <e^{-\alpha t}\left(\varepsilon+M e^{-(L+\alpha) T} \varepsilon / M+M\|x\|\right) \\
& \leq e^{-\alpha t}(2 \varepsilon+M\|x\|) .
\end{aligned}
$$

This shows that the set $\left\{u_{1}, \ldots, u_{N}\right\}$ is $(T, 2 \varepsilon, M, \alpha)$-spanning.

Copyright (c) by SIAM. Unauthorized reproduction of this article is prohibited. 
In summary, we have shown that the minimal cardinality $s_{\text {stab }}(T, 2 \varepsilon, M, \alpha)$ of a spanning set is bounded above by the minimal cardinality of a cover $\mathcal{C}$ of $K$ by $\left(e^{-(L+\alpha) T} \varepsilon / M\right)$-balls. Thus, denoting the minimal cardinality of a cover of a subset $A$ of $\mathbb{R}^{d}$ by $\delta$-balls by $c(\delta, A)$, one has

$$
s_{\text {stab }}(T, 2 \varepsilon, M, \alpha) \leq c\left(e^{-(L+\alpha) T} \varepsilon / M, K\right) .
$$

We want to use that the upper box dimension of a set $A \subset \mathbb{R}^{d}$ is

$$
\limsup _{\delta \searrow 0} \frac{\ln c(\delta, A)}{\ln (1 / \delta)} \leq d
$$

The equality $\ln \left(e^{(L+\alpha) T} M / \varepsilon\right)=(L+\alpha) T+\ln (M / \varepsilon)$ shows that

$$
(L+\alpha) T=\ln \left(e^{(L+\alpha) T} M / \varepsilon\right)-\ln (\varepsilon / M) .
$$

With $\delta=e^{-(L+\alpha) T} \varepsilon / M$ it follows that

$$
\begin{aligned}
\varlimsup_{T \rightarrow \infty} \frac{1}{T} \ln s_{\text {stab }}(T, 2 \varepsilon, M, \alpha) & \leq \varlimsup_{T \rightarrow \infty} \frac{1}{T} \ln c(\delta, K) \\
& =(L+\alpha) \varlimsup_{T \rightarrow \infty} \frac{\ln c(\delta, K)}{(L+\alpha) T} \\
& =(L+\alpha) \varlimsup_{T \rightarrow \infty} \frac{\ln c\left(e^{-(L+\alpha) T} \varepsilon / M, K\right)}{\ln \left(e^{(L+\alpha) T} M / \varepsilon\right)-\ln (\varepsilon / M)} \\
& =(L+\alpha) \varlimsup_{T \rightarrow \infty} \frac{\ln c\left(e^{-(L+\alpha) T} \varepsilon / M, K\right)}{\ln \left(e^{(L+\alpha) T} M / \varepsilon\right)} \\
& =(L+\alpha) \varlimsup_{\delta \searrow 0} \frac{\ln c(\delta, K)}{\ln (1 / \delta)} \leq(L+\alpha) d .
\end{aligned}
$$

For the last inequality, we have used (3.12). Now the assertion follows by letting $\varepsilon$ tend to 0 .

4. Entropy for linear control systems. In this section, the stabilization entropy is determined for linear control systems in $\mathbb{R}^{d}$ of the form

$$
\dot{x}(t)=A x(t)+B u(t), \quad u \in \mathcal{U},
$$

with matrices $A \in \mathbb{R}^{d \times d}$ and $B \in \mathbb{R}^{d \times m}$ and control range $U \subset \mathbb{R}^{m}$ containing the origin. Naturally, the solutions of (4.1) are given by the variation-of-constants formula

$$
\varphi(t, x, u)=e^{A t} x+\int_{0}^{t} e^{A(t-\tau)} B u(\tau) d \tau .
$$

First we prove the following lemma.

LEMma 4.1. Consider a linear control system of the form (4.1) with controls $v$ in a set $\mathcal{V}$ as defined in (3.2). Let $K \subset \mathbb{R}^{d}$ be a compact set with nonvoid interior, and suppose that the eigenvalues $\lambda_{1}, \ldots, \lambda_{d}$ of $A$ counted according to their multiplicity can be ordered such that

$$
\operatorname{Re} \lambda_{1} \leq \operatorname{Re} \lambda_{2} \leq \cdots \leq \operatorname{Re} \lambda_{\ell-1}<0<\operatorname{Re} \lambda_{\ell} \leq \cdots \leq \operatorname{Re} \lambda_{d}
$$

Copyright (C) by SIAM. Unauthorized reproduction of this article is prohibited. 
Take $\alpha$ with $0<\alpha \leq-\operatorname{Re} \lambda_{\ell-1}$ and, if there is no eigenvalue with negative real part, take any $\alpha>0$. Then the $(\alpha, M)$-stabilization entropy of system (4.1) with respect to $K$ satisfies

$$
h_{\mathrm{stab}}(\alpha, M) \geq \sum_{i \geq \ell}\left(\alpha+\operatorname{Re} \lambda_{i}\right) .
$$

Proof. Step 1. Suppose that $\operatorname{Re} \lambda_{i} \geq 0$ for all $i \in\{1, \ldots, d\}$. Then the inequality

$$
h_{\text {stab }}(\alpha, M) \geq \sum_{i=1}^{d}\left(\alpha+\operatorname{Re} \lambda_{i}\right) .
$$

is an immediate consequence of Theorem 3.2 with $f(x, v)=A x+B v$. Here

$$
\operatorname{div}_{x} f(x, v)=\operatorname{tr} \frac{\partial f}{\partial x}(x, v)=\operatorname{tr} A=\sum_{i=1}^{d} \lambda_{i}=\sum_{i=1}^{d} \operatorname{Re} \lambda_{i} .
$$

Step 2. We prove the inequality $h_{\mathrm{stab}}(\alpha, M) \geq \sum_{i \geq \ell}\left(\alpha+\operatorname{Re} \lambda_{i}\right)$ for arbitrary matrices $A$. If all eigenvalues of $A$ have negative real parts, the assertion is true, since $h_{\text {stab }}(\alpha, M, K) \geq 0$ holds anyway. Hence, we may assume that there exists at least one eigenvalue with positive real part. We write $\mathbb{E}^{+}$and $\mathbb{E}^{-}$for the corresponding unstable and stable subspaces, respectively, of the induced flow $(t, x) \mapsto e^{A t} x$. They correspond to the eigenvalues with positive and negative real parts, respectively. This furnishes the decomposition $\mathbb{R}^{d}=\mathbb{E}^{+} \oplus \mathbb{E}^{-}$with associated projection $\pi: \mathbb{R}^{d} \rightarrow \mathbb{E}^{+}$. In an appropriate norm, one has $\|\pi x\| \leq\|x\|$ for all $x \in \mathbb{R}^{d}$. The map $\pi$ is open, hence $\pi(K)$ has nonvoid interior in $\mathbb{E}^{+}$. We can project our control system to $\mathbb{E}^{+}$by

$$
\dot{y}=\pi A y+\pi B v=A \pi x+\pi B v .
$$

One finds that the corresponding trajectories $\pi \varphi$ satisfy the semiconjugation property

$$
(\pi \varphi)(t, \pi(x), v)=\pi(\varphi(t, x, v)) \text { for all } x \in \mathbb{R}^{d}, v \in \mathcal{V}, t \geq 0 .
$$

In particular, $(\pi \varphi)(t, \pi(x), v)=\varphi(t, \pi(x), v))$. Now let $\varepsilon>0$ and $T>0$. Consider a minimal $(T, \varepsilon, \alpha, M)$-spanning set $\mathcal{S} \subset \mathcal{V}$ for $K$ and let $y=\pi(x) \in \pi(K)$ with $x \in K$. Then there is $v \in \mathcal{S}$ such that for all $t \in[0, T]$

$$
\|(\pi \varphi)(t, y, v)\|=\|\pi(\varphi(t, x, v))\|=\|\varphi(t, \pi(x), v)\| \leq e^{-\alpha t}(\varepsilon+M\|y\|) .
$$

This shows that $\mathcal{S}$ is also $(T, \varepsilon, \alpha, M)$-spanning for the set $\pi(K)$ in the projected system. Consequently, taking the limit superior for $T \rightarrow \infty$ and then letting $\varepsilon \searrow 0$, the corresponding entropies satisfy

$$
\begin{aligned}
h_{\text {stab }}(\alpha, M, \pi(K)) & =\lim _{\varepsilon \searrow 0} \varlimsup_{T \rightarrow \infty} \frac{1}{T} \ln s_{\text {stab }}(T, \varepsilon, \alpha, M, \pi(K)) \\
& \leq \lim _{\varepsilon \searrow 0} \varlimsup_{T \rightarrow \infty} \frac{1}{T} \ln s_{\text {stab }}(T, \varepsilon, \alpha, M, K)=h_{\text {stab }}(\alpha, M, K) .
\end{aligned}
$$

The projected system on $\mathbb{E}^{+}$is a linear control system on $\mathbb{E}^{+}$with $\operatorname{dim} \mathbb{E}^{+}=d-\ell+1$, and the eigenvalues $\lambda_{\ell}, \ldots, \lambda_{d}$ of $\left.A\right|_{\mathbb{E}^{-}}: \mathbb{E}^{+} \rightarrow \mathbb{E}^{+}$have positive real parts. Hence we obtain by Step 1 that

$$
h_{\text {stab }}(\alpha, M, K) \geq h_{\text {stab }}(\alpha, M, \pi(K)) \geq(d-\ell+1) \sum_{i \geq \ell} \operatorname{Re} \lambda_{i}=\sum_{i \geq \ell}\left(\alpha+\operatorname{Re} \lambda_{i}\right) .
$$

Copyright @ by SIAM. Unauthorized reproduction of this article is prohibited. 
In order to improve the estimate from above in Theorem 3.3, we will use in an essential way the topological entropy of the linear flow $\Phi_{t} x:=e^{A t} x,(t, x) \in \mathbb{R} \times \mathbb{R}^{d}$. For background on topological entropy in compact metric spaces we refer to Katok and Hasselblatt [10], Robinson [18], or Downarowicz [7]. Topological entropy for linear flows has been characterized by Bowen [1]; see also Walters [21, Theorem 8.14] or Matveev and Savkin [14, Theorem 2.4.2], who write down details for linear maps.

For a compact subset $K \subset \mathbb{R}^{d}$ and $T, \varepsilon>0$, a $(T, \varepsilon, K, \Phi)$-spanning set $S$ is a subset of $\mathbb{R}^{d}$ such that for every $x \in K$ there is $x_{0} \in S$ such that for all $t \in[0, T]$ one has $\left\|e^{A t} x-e^{A t} x_{0}\right\|<\varepsilon$. (Note that here, for the flow, spanning sets are subsets of the state space, while for control systems, we have defined spanning sets as sets of control functions.) Then, denoting by $s_{\text {top }}(T, \varepsilon, K, \Phi)$ the minimal cardinality of a $(T, \varepsilon, K, \Phi)$-spanning set, the topological entropy of $K$ is defined as

$$
h_{\mathrm{top}}(K, \Phi)=\lim _{\varepsilon \searrow 0} \varlimsup_{T \rightarrow \infty} \frac{1}{T} \ln s_{\mathrm{top}}(T, \varepsilon, K, \Phi) .
$$

Finally, the topological entropy is defined as the supremum over compact sets $K \subset \mathbb{R}^{d}$, and it is equal to the sum of the positive real parts of the eigenvalues of $A$,

$$
h_{\mathrm{top}}(\Phi):=\sup _{K} h_{\mathrm{top}}(K, \Phi)=\sum_{\operatorname{Re} \lambda_{i}>0} \operatorname{Re} \lambda_{i} .
$$

An equivalent definition of topological entropy is obtained, when one replaces $s_{\text {top }}(T, \varepsilon, K, \Phi)$ by the maximal cardinality of a $(T, \varepsilon, K, \Phi)$-separated set, which is a subset $E$ of $K$ such that for all $x_{0}, x_{1} \in E$ there is $t \in[0, T]$ with $\left\|e^{A t} x_{0}-e^{A t} x_{1}\right\|>\varepsilon$.

The next theorem characterizes the stabilization entropy regarding the equilibrium $x^{*}=0$ for linear control systems.

TheOREM 4.2. Consider a linear control system of the form (4.1) with $0 \in U$. Assume that there are $M, \alpha>0$ such that for all initial values $0 \neq x \in \mathbb{R}^{d}$ there is a control $u \in \mathcal{U}$ with

$$
\|\varphi(t, x, u)\|<M e^{-\alpha t}\|x\| \text { for all } t \geq 0 .
$$

For every compact set $K \subset \mathbb{R}^{d}$ the $(\alpha, M)$-stabilization entropy of system (4.1) satisfies

$$
h_{\text {stab }}(\alpha, M, K) \leq \sum_{\operatorname{Re} \lambda_{i}>-\alpha}\left(\alpha+\operatorname{Re} \lambda_{i}\right) ;
$$

here summation is over all eigenvalues $\lambda_{i}$ of $A$, counted according to their multiplicity with $\operatorname{Re} \lambda_{i}>-\alpha$.

If, additionally, the set $K$ has nonvoid interior in $\mathbb{R}^{d}$, equality holds in (4.4). In particular, $h_{\mathrm{stab}}(\alpha, M, K)$ is independent of $K$ in this situation.

Proof. The proof is divided into three steps.

Step 1. We show that $h_{\text {stab }}(\alpha, M, K) \leq \sum_{\operatorname{Re} \lambda_{i}>-\alpha}\left(\alpha+\operatorname{Re} \lambda_{i}\right)$ using Bowen's result for topological entropy of linear flows. Order the eigenvalues $\lambda_{1}, \ldots, \lambda_{d}$ of $A$ such that

$$
\operatorname{Re} \lambda_{1} \leq \cdots \leq \operatorname{Re} \lambda_{d}
$$

Let $\alpha \in\left(-\operatorname{Re} \lambda_{\ell},-\operatorname{Re} \lambda_{\ell-1}\right]$ with $\operatorname{Re} \lambda_{\ell} \leq 0$. Then the eigenvalues of $A+\alpha I$ with positive real parts are given by $\alpha+\operatorname{Re} \lambda_{\ell}, \ldots, \alpha+\operatorname{Re} \lambda_{d}$, and hence the topological entropy of the flow $\Phi_{t}^{\alpha}=e^{(A+\alpha I) t}=e^{\alpha t} e^{A t}, t \in \mathbb{R}$, is given by

$$
h_{\mathrm{top}}\left(\Phi^{\alpha}\right)=\sum_{\operatorname{Re} \lambda_{i}>-\alpha}\left(\alpha+\operatorname{Re} \lambda_{i}\right) .
$$


If $\alpha>-\operatorname{Re} \lambda_{1}$, all eigenvalues of $A+\alpha I$ have positive real parts, and if $\operatorname{Re} \lambda_{d}<0$ and $\alpha \in\left(0,-\operatorname{Re} \lambda_{d}\right)$, there is no eigenvalue of $A+\alpha I$ with positive real part.

Fix $\varepsilon>0$ and $T>0$. Let $E \subset K$ be a maximal $\left(T, \varepsilon, K, \Phi^{\alpha}\right)$-separated set, say $E=\left\{x_{1}, \ldots, x_{k}\right\}$ with cardinality $k=s_{\text {top }}\left(T, \varepsilon, K, \Phi^{\alpha}\right)$. Without loss of generality, we may take $x_{j} \neq 0$ for all $j$. Then $E$ is also $\left(T, \varepsilon, K, \Phi^{\alpha}\right)$-spanning, which means that for all $x \in K$ there is $x_{j} \in E$ such that for all $t \in[0, T]$

$$
\left\|e^{(A+\alpha I) t} x-e^{(A+\alpha I) t} x_{j}\right\|<\varepsilon \text {, i.e., }\left\|e^{A t} x-e^{A t} x_{j}\right\|<e^{-\alpha t} \varepsilon .
$$

By assumption (4.3), we can assign to each $x_{j} \in E \subset K, j=1, \ldots, k$, a control function $u_{j} \in \mathcal{U}$ such that

$$
\left\|\varphi\left(t, x_{j}, u_{j}\right)\right\|<M e^{-\alpha t}\left\|x_{j}\right\| \text { for all } t \geq 0
$$

Let $\mathcal{S}:=\left\{u_{1}, \ldots, u_{k}\right\} \subset \mathcal{U}$. Using linearity, we obtain that for all $x \in K$ there is $j$ such that for all $t \in[0, T]$

$$
\left\|\varphi\left(t, x, u_{j}\right)-\varphi\left(t, x_{j}, u_{j}\right)\right\|=\left\|e^{A t} x-e^{A t} x_{j}\right\|<e^{-\alpha t} \varepsilon
$$

and hence

$$
\begin{aligned}
\left\|\varphi\left(t, x, u_{j}\right)\right\| & \leq\left\|\varphi\left(t, x, u_{j}\right)-\varphi\left(t, x_{j}, u_{j}\right)\right\|+\left\|\varphi\left(t, x_{j}, u_{j}\right)\right\| \\
& <\varepsilon e^{-\alpha t}+M e^{-\alpha t}\left\|x_{j}\right\| \\
& \leq \varepsilon e^{-\alpha t}+M e^{-\alpha t}\left\|x_{j}-x\right\|+M e^{-\alpha t}\|x\| \\
& \leq e^{-\alpha t}[\varepsilon(1+M)+M\|x\|] .
\end{aligned}
$$

This shows that the set $\mathcal{S}$ is $(T, \varepsilon(1+M), \alpha, M)$-spanning. It follows that for all $T>0, \varepsilon>0$

$$
s_{\mathrm{stab}}(T, \varepsilon(1+M), M, \alpha) \leq k=s_{\mathrm{top}}\left(T, \varepsilon, K, \Phi^{\alpha}\right),
$$

and consequently

$$
\begin{aligned}
h_{\text {stab }}(\alpha, M, K) & =\lim _{\varepsilon \searrow 0} \varlimsup_{T \rightarrow \infty} \frac{1}{T} \ln s_{\text {stab }}(T, \varepsilon, \alpha, M) \\
& =\lim _{\varepsilon \searrow 0} \varlimsup_{T \rightarrow \infty} \frac{1}{T} \ln s_{\text {stab }}(T, \varepsilon(1+M), M, \alpha) \\
& \leq \lim _{\varepsilon \searrow 0} \varlimsup_{T \rightarrow \infty} \frac{1}{T} \ln s_{\text {top }}\left(T, \varepsilon, K, \Phi^{\alpha}\right) \\
& =h_{\text {top }}\left(K, \Phi^{\alpha}\right) \\
& \leq h_{\text {top }}\left(\Phi^{\alpha}\right)=\sum_{i \geq \ell}\left(\alpha+\operatorname{Re} \lambda_{i}\right) .
\end{aligned}
$$

Step 2. In order to prove the converse inequality, consider for $\beta \in(0, \alpha)$ the control system

$$
\begin{aligned}
& \dot{y}=(A+\beta I) y+B v(t), \\
& v \in \mathcal{V}(\beta):=\left\{v: \mathbb{R} \rightarrow \mathbb{R}^{m} \mid v(t)=e^{\beta t} u(t), t \in \mathbb{R} \text { for some } u \in \mathcal{U}\right\}
\end{aligned}
$$

Copyright ( by SIAM. Unauthorized reproduction of this article is prohibited. 
Then the solutions $\psi\left(t, x_{0}, v\right)$ of (4.5) and the solutions of (4.1) are related by

$$
\begin{aligned}
\psi\left(t, x_{0}, v\right) & =e^{(A+\beta I) t} x_{0}+\int_{0}^{t} e^{(A+\beta I)(t-\tau)} B v(\tau) d \tau \\
& =e^{\beta t} e^{A t} x_{0}+\int_{0}^{t} e^{A(t-\tau)} B e^{\beta(t-\tau)} v(\tau) d \tau \\
& =e^{\beta t}\left[e^{A t} x_{0}+\int_{0}^{t} e^{A(t-\tau)} B e^{-\beta \tau} v(\tau) d \tau\right] \\
& =e^{\beta t}\left[e^{A t} x_{0}+\int_{0}^{t} e^{A(t-\tau)} B u(\tau) d \tau\right] \\
& =e^{\beta t} \varphi\left(t, x_{0}, u\right)
\end{aligned}
$$

with $u(t):=e^{-\beta t} v(t), t \geq 0$.

Now let $\varepsilon>0$ and $T>0$. Consider a minimal $(T, \varepsilon, \alpha, M)$-spanning set $\mathcal{S}=$ $\left\{u_{1}, \ldots, u_{k}\right\} \subset \mathcal{U}$ of $(4.1)$ and define $v_{i} \in \mathcal{V}(\beta)$ by $v_{i}(t):=e^{\beta t} u(t), t \geq 0$. Then for every $x_{0} \in K$ there is $u_{i} \in \mathcal{S}$ such that for $[0, T]$

$$
\left\|\psi\left(t, x_{0}, v_{i}\right)\right\|=e^{\beta t}\left\|\varphi\left(t, x_{0}, u_{i}\right)\right\|<e^{-(\alpha-\beta) t}\left(\varepsilon+M\left\|x_{0}\right\|\right) .
$$

This shows that $\left\{v_{1}, \ldots, v_{k}\right\} \subset \mathcal{V}(\beta)$ is $(T, \varepsilon, \alpha-\beta, M)$-spanning for system (4.5). Consequently, the stabilization entropy $h_{\text {stab }}(\alpha, M, K)$ of system (4.1) is bounded below by the $(\alpha-\beta)$-stabilization entropy of system (4.5).

Step 3. Let $\alpha, \beta>0$ such that $\operatorname{Re} \lambda_{\ell-1} \leq-\alpha<-\beta<\operatorname{Re} \lambda_{\ell}$. We will conclude the proof by showing that for system (4.5)

$$
h_{\mathrm{stab}}(\alpha-\beta, K) \geq \sum_{i \geq \ell}\left(\alpha+\operatorname{Re} \lambda_{i}\right) .
$$

This follows by an application of Lemma 4.1. The assumptions of this lemma hold, since by choice of $\beta$ the eigenvalues of $A+\beta I$ with positive real parts are given by $\beta+\operatorname{Re} \lambda_{\ell}, \ldots, \beta+\operatorname{Re} \lambda_{d}$ and the largest eigenvalue with negative real part is $\beta+\operatorname{Re} \lambda_{\ell-1}$. Furthermore, since $\alpha \leq-\operatorname{Re} \lambda_{\ell-1}$, one has

$$
0<\alpha-\beta \leq-\left(\beta+\operatorname{Re} \lambda_{\ell-1}\right) .
$$

Lemma 4.1 implies that the stabilization entropy of system (4.5) is

$$
h_{\mathrm{stab}}(\alpha-\beta, K) \geq \sum_{i \geq \ell}\left[(\alpha-\beta)+\left(\beta+\operatorname{Re} \lambda_{i}\right)\right]=\sum_{i \geq \ell}\left(\alpha+\operatorname{Re} \lambda_{i}\right) .
$$

For $\ell=1$ and $\ell=d$, one argues analogously. This completes the proof of the theorem.

Remark 4.3. For smooth nonlinear control systems and arbitrarily small control range, we conjecture that a formula analogous to (4.4) now holds for the Jacobian at the equilibrium. Compare Nair et al. [17, Theorem 3] for such a claim in the context of local uniform asymptotic stabilization.

Remark 4.4. In a discrete time setting, a formula similar to (4.4) shows up in Nair and Evans [15, Theorem 1] for a problem with random initial states.

Copyright $@$ by SIAM. Unauthorized reproduction of this article is prohibited. 
5. Minimal bit rates for stabilization. In this section, the minimal bit rate for stabilization is related to the stabilization entropy.

Recall that for the definition of the stabilization entropy $h_{\mathrm{stab}}(\alpha, M, K)$ only controls on bounded intervals of the form $[0, T]$ are employed; see (2.9). Hence we have to concatenate controls defined on finite intervals in order to obtain controls defined for all $t \geq 0$ as needed for bit rates. This is prepared by the following key technical lemma.

Lemma 5.1. Consider a compact neighborhood $K$ of the equilibrium $x^{*}=0$ for system (2.1). Let $M>1$ and $\alpha^{*}>\alpha>0$ and suppose that $\varepsilon \in\left(0, \frac{1}{2}\right)$ is small enough such that $K$ contains the $2 \varepsilon$-neighborhood of the equilibrium. Consider a sequence of times $T_{n}>T:=T_{1}>T_{0}:=0, n \in \mathbb{N}$, such that there is a $\left(T_{n}, \varepsilon^{n+2}, \alpha^{*}, M\right)$-spanning set $\mathcal{S}_{n} \subset \mathcal{U}$ of controls with a minimal number $s_{n}=s_{\text {stab }}\left(T_{n}, \varepsilon^{n+2}, \alpha^{*}, M\right) \in \mathbb{N}$ of elements and $T_{n}$ is large enough such that

$$
e^{-\alpha^{*} T_{n}} M<\frac{\varepsilon^{n+2}}{3}, T_{n}>\frac{\ln M}{\alpha^{*}-\alpha} \text { and } e^{-\alpha T_{n}} M \max _{x \in K}\|x\|<\frac{\varepsilon^{n+2}}{2} .
$$

Abbreviate $\tau_{n}:=\sum_{i=0}^{n} T_{i}, n \geq 0$. Define a set $\mathcal{R}^{*} \subset \mathcal{U}$ in the following way: $v \in \mathcal{R}^{*}$ if for all $n \geq 1$ there is $u_{n} \in \mathcal{S}_{n}$ with

$$
v(t)=u_{n}\left(t-\tau_{n-1}\right) \text { for } t \in\left(\tau_{n-1}, \tau_{n}\right] .
$$

Then for every $x \in K$ there is a control $v \in \mathcal{R}^{*}$ with

$$
\left\|\varphi\left(\tau_{n}, x, v\right)\right\|<2 \varepsilon \text { for all } n \geq 1
$$

and

$$
\|\varphi(t, x, v)\|<2 \varepsilon^{n+2}+M e^{-\alpha t}\|x\| \text { for all } t \in\left[\tau_{n}, \tau_{n+1}\right] \text { and } n \geq 0 .
$$

Proof. The first inequality in (5.1) and $\varepsilon \in(0,1)$ imply $M e^{-\alpha^{*} T_{n}}<\frac{\varepsilon^{n+2}}{3}<\frac{1}{3}$, and hence using $T_{n}>T$

$$
\frac{1}{1-\frac{M e^{-\alpha^{*} T_{n}}}{\varepsilon^{n+2}}}<\frac{1}{1-M e^{-\alpha^{*} T}}<\frac{3}{2} .
$$

Furthermore, the second inequality in (5.1) implies $T_{n}\left(\alpha^{*}-\alpha\right)>\ln M$ and hence $e^{\alpha^{*} T_{n}}>e^{\alpha T_{n}} M$ and, finally,

$$
M e^{-\alpha^{*} T_{n}}<e^{-\alpha T_{n}}<1 .
$$

By definition of $\mathcal{S}_{n}$, for every $x \in K$ there exists $u_{n} \in \mathcal{S}_{n}$ such that for all $t \in\left[0, T_{n}\right]$

$$
\left\|\varphi\left(t, x, u_{n}\right)\right\|<e^{-\alpha^{*} t}\left(\varepsilon^{n+2}+M\|x\|\right) .
$$

In particular, for every $x \in K$ there exists $v_{1}:=u_{1} \in \mathcal{S}_{1}$ such that for all $t \in\left[0, T_{1}\right]$

$$
\left\|\varphi\left(t, x, v_{1}\right)\right\|<e^{-\alpha^{*} t}\left(\varepsilon^{2}+M\|x\|\right) \leq \varepsilon^{2}+M e^{-\alpha^{*} t}\|x\|,
$$

which implies assertion (5.3) for $n=1$. By the third inequality in (5.1)

$$
\left\|\varphi\left(T_{1}, x, v_{1}\right)\right\|<\varepsilon^{2}+M e^{-\alpha^{*} T_{1}}\|x\|<2 \varepsilon,
$$

and it follows that $\varphi\left(T_{1}, x, v_{1}\right) \in K$. 
Now we fix $x \in K$ and define inductively controls $v_{n} \in \mathcal{U}$ with the following properties: For all $n \geq 1$ one has

$$
\varphi\left(\tau_{n}, x, v_{n}\right) \in K \text { and }\left\|\varphi\left(\tau_{n}, x, v_{n}\right)\right\| \leq \sum_{i=0}^{n-1}\left(M e^{-\alpha^{*} T}\right)^{i} \varepsilon+\prod_{i=1}^{n}\left(M e^{-\alpha^{*} T_{i}}\right)\|x\| .
$$

By (5.8) the control $v_{1}$ satisfies (5.9) for $n=1$. Suppose that (5.9) holds for $n$. Then, by (5.6), there is a control $u_{n+1} \in \mathcal{S}_{n+1}$ such that for $t \in\left[0, T_{n+1}\right]$

$$
\left\|\varphi\left(t, \varphi\left(\tau_{n}, x, v_{n}\right), u_{n+1}\right)\right\|<e^{-\alpha^{*} t}\left(\varepsilon^{n+2}+M\left\|\varphi\left(\tau_{n}, x, v_{n}\right)\right\|\right) .
$$

Define the control $v_{n+1}$ by

$$
v_{n+1}(t):=\left\{\begin{array}{cl}
v_{n}(t) & \text { for } \quad t \in\left[0, \tau_{n}\right], \\
u_{n+1}\left(t-\tau_{n}\right) & \text { for } \quad t \in\left(\tau_{n}, \tau_{n+1}\right] .
\end{array}\right.
$$

Then by the induction hypothesis (5.9) for $n$ and $T_{n} \geq T$

$$
\begin{aligned}
\left\|\varphi\left(\tau_{n+1}, x, v_{n+1}\right)\right\| & \left.=\| \varphi\left(T_{n+1}, \varphi\left(\tau_{n}, x, v_{n}\right), u_{n+1}\right)\right) \| \\
& <e^{-\alpha^{*} T_{n+1}}\left(\varepsilon^{n+2}+M\left\|\varphi\left(\tau_{n}, x, v_{n}\right)\right\|\right) \\
& <\varepsilon+M e^{-\alpha^{*} T_{n+1}}\left[\sum_{i=0}^{n-1}\left(M e^{-\alpha^{*} T}\right)^{i} \varepsilon+\prod_{i=1}^{n}\left(M e^{-\alpha^{*} T_{i}}\right)\|x\|\right] \\
& \leq \varepsilon+M e^{-\alpha^{*} T} \sum_{i=0}^{n-1}\left(M e^{-\alpha^{*} T}\right)^{i} \varepsilon+M e^{-\alpha^{*} T_{n+1}} \prod_{i=1}^{n}\left(M e^{-\alpha^{*} T_{i}}\right)\|x\| \\
& =\sum_{i=0}^{n}\left(M e^{-\alpha^{*} T}\right)^{i} \varepsilon+\prod_{i=1}^{n+1}\left(M e^{-\alpha^{*} T_{i}}\right)\|x\|<\frac{3}{2} \varepsilon+\frac{\varepsilon}{2}=2 \varepsilon .
\end{aligned}
$$

In the last line we have also used (5.4) and the last inequality in (5.1). This concludes the induction step, since it shows that (5.9) also holds for $n+1$ instead of $n$. The control $v \in \mathcal{R}^{*}$ coinciding on $\left[0, \tau_{n}\right]$ with $v_{n}, n \in \mathbb{N}$, is well defined, and assertion (5.2) is verified.

Next we prove inequality (5.3). It holds on $\left[0, T_{1}\right]$, i.e., for $n=0$, by (5.7). We claim that for all $n \geq 1$

$$
\left\|\varphi\left(\tau_{n}, x, v_{n}\right)\right\| \leq \sum_{i=0}^{n-1} M^{i}\left(e^{-\alpha^{*} T}\right)^{i+1} \varepsilon^{n-i+1}+\prod_{i=1}^{n}\left(M e^{-\alpha^{*} T_{i}}\right)\|x\| .
$$

By (5.7) this holds for $n=1$, since

$$
\left\|\varphi\left(T_{1}, x, u_{1}\right)\right\|<e^{-\alpha^{*} T} \varepsilon^{2}+M e^{-\alpha^{*} T_{1}}\|x\| .
$$

Again arguing inductively, suppose that (5.11) holds for $n$. By (5.10) and the induction hypothesis, the control $v_{n+1}$ satisfies for $t \in\left[\tau_{n}, \tau_{n+1}\right]$

$$
\begin{aligned}
& \left\|\varphi\left(t, x, v_{n+1}\right)\right\| \\
& \quad=\left\|\varphi\left(t-\tau_{n}, \varphi\left(\tau_{n}, x, v_{n}\right), u_{n+1}\right)\right\| \\
& \quad<e^{-\alpha^{*}\left(t-\tau_{n i}\right)}\left(\varepsilon^{n+2}+M\left\|\varphi\left(\tau_{n}, x, v_{n}\right)\right\|\right) \\
& \quad \leq e^{-\alpha^{*}\left(t-\tau_{n}\right)}\left(\varepsilon^{n+2}+M \sum_{i=0}^{n-1} M^{i}\left(e^{-\alpha^{*} T}\right)^{i+1} \varepsilon^{n-i+1}+M \prod_{i=1}^{n}\left(M e^{-\alpha^{*} T_{i}}\right)\|x\|\right),
\end{aligned}
$$


and for $t=\tau_{n+1}$ we obtain assertion (5.11) for $n+1$, since

$$
\begin{aligned}
& \left\|\varphi\left(\tau_{n+1}, x, v_{n+1}\right)\right\| \\
& <e^{-\alpha^{*} T_{n+1}} \varepsilon^{n+2}+M e^{-\alpha^{*} T_{n+1}} \sum_{i=0}^{n-1} M^{i}\left(e^{-\alpha^{*} T}\right)^{i+1} \varepsilon^{n-i+1}+\prod_{i=1}^{n+1}\left(M e^{-\alpha^{*} T_{i}}\right)\|x\| \\
& <e^{-\alpha^{*} T} \varepsilon^{n+2}+\sum_{i=0}^{n-1} M^{i+1}\left(e^{-\alpha^{*} T}\right)^{i+2} \varepsilon^{n+1-i}+\prod_{i=1}^{n+1}\left(M e^{-\alpha^{*} T_{i}}\right)\|x\| \\
& =\sum_{i=0}^{n} M^{i}\left(e^{-\alpha^{*} T}\right)^{i+1} \varepsilon^{n+2-i}+\prod_{i=1}^{n+1}\left(M e^{-\alpha^{*} T_{i}}\right)\|x\| .
\end{aligned}
$$

Thus assertion (5.11) holds for all $n$. For $t \in\left[\tau_{n}, \tau_{n+1}\right], n \geq 0$, inequalities (5.12) and (5.11), and, finally, (5.4) yield

$$
\begin{aligned}
& \left\|\varphi\left(t, x, v_{n+1}\right)\right\| \\
& \leq \varepsilon^{n+2}+M \sum_{i=0}^{n-1} M^{i}\left(e^{-\alpha^{*} T}\right)^{i+1} \varepsilon^{n-i+1}+e^{-\alpha^{*}\left(t-\tau_{n}\right)} M \prod_{i=1}^{n}\left(M e^{-\alpha^{*} T_{i}}\right)\|x\| \\
& \leq \varepsilon^{n+2}\left[\sum_{i=0}^{n}\left(\frac{M e^{-\alpha^{*} T}}{\varepsilon}\right)^{i}\right]+e^{-\alpha\left(t-\tau_{n}\right)} M \prod_{i=1}^{n}\left(M e^{-\alpha^{*} T_{i}}\right)\|x\| .
\end{aligned}
$$

By (5.5) $M e^{-\alpha^{*} T_{n}}<e^{-\alpha T_{n}}$, and (5.3) follows, since for $t \in\left[\tau_{n}, \tau_{n+1}\right]$

$$
\left\|\varphi\left(t, x, v_{n+1}\right)\right\|<\frac{3}{2} \varepsilon^{n+2}+e^{-\alpha\left(t-\tau_{n}\right)} M \prod_{i=1}^{n} e^{-\alpha T_{i}}\|x\|<2 \varepsilon^{n+2}+M e^{-\alpha t}\|x\| .
$$

An easy consequence is the following observation which shows that we have constructed a stabilizing set of control functions.

LEMMA 5.2. Under the assumption of Lemma 5.1, define an $\mathcal{L}_{\varepsilon}$-function $\gamma$ by

$$
\gamma(t):=\varepsilon^{n+1} \text { for all } t \in\left[\tau_{n}, \tau_{n+1}\right) \text { and } n \geq 0 \text {. }
$$

Then the set $\mathcal{R}^{*}$ is $(\gamma, \varepsilon, \alpha, M)$-stabilizing, i.e., it is a set of the form $\mathcal{R}(\gamma, \varepsilon, \alpha, M)$ with the property that for every $x \in K$ there is $v \in \mathcal{R}^{*}$ with

$$
\|\varphi(t, x, v)\|<\gamma(t)+M e^{-\alpha t}\|x\| \text { for } t \geq 0
$$

Proof. Note that $2 \varepsilon<1$. Assertion (5.13) follows from inequality (5.3) showing that for $t \in\left[\tau_{n}, \tau_{n+1}\right), n \geq 0$,

$$
\|\varphi(t, x, v)\|<2 \varepsilon^{n+2}+M e^{-\alpha t}\|x\| s<\varepsilon^{n+1}+M e^{-\alpha t}\|x\| .
$$

The next theorem establishes the announced relation between the stabilization bit rate and the stabilization entropy.

THEOREM 5.3. Consider a control system of the form (2.1) and suppose that $K \subset \mathbb{R}^{d}$ is a compact neighborhood of the equilibrium $x^{*}=0$. Assume that there are constants $M>1$ and $\alpha^{*}>0$ such that for all $0 \neq x \in K$ there is $u \in \mathcal{U}$ with

$$
\|\varphi(t, x, u)\|<M e^{-\alpha^{*} t}\|x\| \text { for all } t \geq 0 .
$$

Copyright (C) by SIAM. Unauthorized reproduction of this article is prohibited. 
Then for $\alpha \in\left(0, \alpha^{*}\right)$ the stabilization bit rate and the stabilization entropy satisfy

$$
b_{\text {stab }}(\alpha, M) \leq \log _{2} e \cdot h_{\text {stab }}\left(\alpha^{*}, M\right)<\infty .
$$

Proof. By Theorem 3.3 the stabilization entropy for $\alpha^{*}$ is finite. Hence the first inequality remains to be shown. In the proof, we will use the natural logarithm also for the bit rate, and at the end we will use the second part of Remark 2.8. Throughout the proof, the constant $M$ remains fixed, so we omit this argument. Let $\varepsilon>0$ and observe that for every $n \geq 0$

$$
\varlimsup_{T^{\prime} \rightarrow \infty} \frac{1}{T^{\prime}} \ln s_{\text {stab }}\left(T^{\prime}, \varepsilon^{n+2}, \alpha^{*}\right) \leq \lim _{\varepsilon^{\prime} \searrow 0} \varlimsup_{T^{\prime} \rightarrow \infty} \frac{1}{T^{\prime}} \ln s_{\text {stab }}\left(T^{\prime}, \varepsilon^{\prime}, \alpha^{*}\right) .
$$

Fix $\delta>0$. Then there are $T_{n}>1$, arbitrarily large, such that

$$
\frac{1}{T_{n}} \ln s_{\text {stab }}\left(T_{n}, \varepsilon^{n+2}, \alpha^{*}\right) \leq \lim _{\varepsilon^{\prime} \backslash 0} \varlimsup_{T^{\prime} \rightarrow \infty} \frac{1}{T^{\prime}} \ln s_{\text {stab }}\left(T^{\prime}, \varepsilon^{\prime}, \alpha^{*}\right)+\delta .
$$

Choose $T_{n}$ large enough such that condition (5.1) in Lemma 5.1 is satisfied. Then hypothesis (5.14) and Lemma 2.3 show that there are finite $\left(T_{n}, \varepsilon^{n+2}, \alpha^{*}\right)$-spanning sets $\mathcal{S}_{n}$ as assumed in Lemma 5.1.

By the construction in Lemma 5.1 there is for every $\alpha \in\left(0, \alpha^{*}\right)$ a set $\mathcal{R}^{*}$ which by Lemma 5.2 may be written as $\mathcal{R}^{*}=\mathcal{R}(\gamma, \varepsilon, \alpha)$. On an interval $\left[\tau_{n-1}, \tau_{n}\right]$ controls in $\mathcal{S}_{n}$ are used, hence their number is at most $s_{n}=s_{\text {stab }}\left(T_{n}, \varepsilon^{n+2}, \alpha^{*}\right)$. Thus the number of restricted controls on $\left[0, \tau_{n}\right], n \geq 1$, is

$$
\# \mathcal{R}_{\tau_{n}}(\gamma, \varepsilon, \alpha)=\prod_{i=1}^{n} s_{\mathrm{stab}}\left(T_{i}, \varepsilon^{i+2}, \alpha^{*}\right) .
$$

Hence one finds

$$
\frac{1}{\tau_{n}} \ln \# \mathcal{R}_{\tau_{n}}(\gamma, \varepsilon, \alpha) \leq \frac{1}{\tau_{n}} \ln \left[\prod_{i=1}^{n} s_{\text {stab }}\left(T_{i}, \varepsilon^{i+2}, \alpha^{*}\right)\right]=\frac{1}{\tau_{n}} \sum_{i=1}^{n} \ln s_{\text {stab }}\left(T_{i}, \varepsilon^{i+2}, \alpha^{*}\right) .
$$

Now observe that the right-hand side is a convex combination of

$$
\frac{1}{T_{i}} \ln s_{\mathrm{stab}}\left(T_{i}, \varepsilon^{i+2}, \alpha^{*}\right), i=1, \ldots, n,
$$

since

$$
\begin{aligned}
& \frac{1}{T_{i}+T_{j}}\left[\ln s_{\text {stab }}\left(T_{i}, \varepsilon^{i+2}, \alpha^{*}\right)+\ln s_{\text {stab }}\left(T_{j}, \varepsilon^{j+2}, \alpha^{*}\right)\right] \\
& \quad=\frac{T_{i}}{T_{i}+T_{j}} \frac{1}{T_{i}} \ln s_{\text {stab }}\left(T_{i}, \varepsilon^{i+2}, \alpha^{*}\right)+\frac{T_{j}}{T_{i}+T_{j}} \frac{1}{T_{j}} \ln s_{\mathrm{stab}}\left(T_{j}, \varepsilon^{j+2}, \alpha^{*}\right) .
\end{aligned}
$$

Together with (5.15) this implies for all $n$

$$
\frac{1}{\tau_{n}} \ln \# \mathcal{R}_{\tau_{n}}(\gamma, \varepsilon, \alpha) \leq \lim _{\varepsilon^{\prime} \backslash 0} \varlimsup_{T^{\prime} \rightarrow \infty} \frac{1}{T^{\prime}} \ln s_{\text {stab }}\left(T^{\prime}, \varepsilon^{\prime}, \alpha^{*}\right)+\delta .
$$

For $n \rightarrow \infty$ this yields

$$
\varliminf_{\tau \rightarrow \infty} \frac{1}{\tau} \ln \# \mathcal{R}_{\tau}(\gamma, \varepsilon, \alpha) \leq \lim _{\varepsilon^{\prime} \searrow 0} \varlimsup_{T^{\prime} \rightarrow \infty} \frac{1}{T^{\prime}} \ln s_{\mathrm{stab}}\left(T^{\prime}, \varepsilon^{\prime}, \alpha^{*}\right)+\delta,
$$


and since $\delta>0$ is arbitrary,

$$
\varliminf_{\tau \rightarrow \infty} \frac{1}{\tau} \ln \# \mathcal{R}_{\tau}(\gamma, \varepsilon, \alpha) \leq \lim _{\varepsilon^{\prime} \searrow 0} \varlimsup_{T^{\prime} \rightarrow \infty} \frac{1}{T^{\prime}} \ln s_{\mathrm{stab}}\left(T^{\prime}, \varepsilon^{\prime}, \alpha^{*}\right)=h_{\mathrm{stab}}\left(\alpha^{*}\right) .
$$

This remains true for the infimum over all $\mathcal{L}_{\varepsilon}$-functions $\gamma$ and then for the limit for $\varepsilon \searrow 0$, and we conclude using Remark 2.8,

$b_{\text {stab }}(\alpha, M)=\log _{2} e \cdot \lim _{\varepsilon \searrow 0} \inf _{\mathcal{R}(\gamma, \varepsilon, \alpha)} \varlimsup_{T^{\prime} \rightarrow \infty} \frac{1}{T} \ln \# \mathcal{R}_{T}(\gamma, \varepsilon, \alpha) \leq \log _{2} e \cdot h_{\text {stab }}\left(\alpha^{*}, M\right)$.

For linear control systems, one gets an easy corollary.

Corollary 5.4. Consider a linear control system of the form (4.1) with $0 \in U$ and let $K$ be a compact neighborhood of the origin such that for some constants $M>1$ and $\alpha^{*}>0$ and all initial values $0 \neq x \in K$ there is a control $u \in \mathcal{U}$ with

$$
\|\varphi(t, x, u)\|<M e^{-\alpha^{*} t}\|x\| \text { for all } t \geq 0 .
$$

Then for all $\alpha \in\left(0, \alpha^{*}\right)$ the stabilization bit rate satisfies

$$
b_{\text {stab }}(\alpha, M) \leq \log _{2} e \cdot h_{\text {stab }}(\alpha, M)=\log _{2} e \cdot \sum_{\operatorname{Re} \lambda_{i}>-\alpha}\left(\alpha+\operatorname{Re} \lambda_{i}\right) .
$$

Proof. Theorem 4.2 implies for all $\alpha \in\left(0, \alpha^{*}\right)$ that the equality in (5.16) holds. In order to show the inequality, choose $\alpha^{\prime}$ with $\alpha<\alpha^{\prime}<\alpha^{*}$ such that the eigenvalues $\lambda$ of $A$ satisfy $\operatorname{Re} \lambda>\alpha$ iff $\operatorname{Re} \lambda>\alpha^{\prime}$. Then $h_{\text {stab }}\left(\alpha^{\prime}, M\right)=h_{\text {stab }}(\alpha, M)$ and Theorem 5.3 implies the inequality.

For small decay rate $\alpha$, we can improve this estimate using asymptotic invariance entropy defined in Colonius and Kawan [3, Definition 5]. We specialize and slightly modify it for our purposes. Consider a linear control system of the form (4.1). Let $K$ be a compact set with nonvoid interior. Fix $\varepsilon>0$ and times $T>T_{0} \geq 0$. A set $\mathcal{S} \subset \mathcal{U}$ is called $\left(T, T_{0}, \varepsilon, K\right)$-spanning if for every $x \in K$ there is $u \in \mathcal{S}$ with

$$
\operatorname{dist}(\varphi(t, x, u), K):=\min \{\|x-y\| \mid y \in K\}<\varepsilon \text { for all } t \in\left[T_{0}, T\right] .
$$

The minimal cardinality of such a set is $r_{\text {as }}\left(T, T_{0}, \varepsilon, K\right)$, and we define the asymptotic invariance entropy as

$$
h_{\mathrm{as}}(K):=\lim _{\varepsilon \searrow 0} \lim _{T_{0} \rightarrow \infty} \underline{\lim }_{T \rightarrow \infty} \frac{1}{T} \ln r_{\mathrm{as}}\left(T, T_{0}, \varepsilon, K\right) .
$$

Then the following result holds.

Proposition 5.5. Consider a linear control system of the form (4.1). Let $K$ be a compact neighborhood of the origin, let $0 \in U$, and suppose that there is $T_{0}>0$ such that for all $x \in K$ there is a control $u \in \mathcal{U}$ with $\varphi(t, x, u) \in K$ for all $t \geq T_{0}$. Then

$$
h_{\mathrm{as}}(K) \geq \sum_{\operatorname{Re} \lambda_{i}>0} \operatorname{Re} \lambda_{i}
$$

where summation is over all eigenvalues of $A$ with $\operatorname{Re} \lambda_{i}>0$.

Proof. This follows similarly as Colonius and Kawan [2, Theorem 5.1]. The differences are that here (i) we consider asymptotic invariance instead of invariance, (ii) we do not assume compactness of the control range, and (iii) the limit inferior for 
$T \rightarrow \infty$ is considered instead of the limit superior. If $A$ has only unstable eigenvalues, [3, Lemma 5], which does not need compactness of the control range $U$, shows that points (i) and (ii) can be dealt with. Concerning (iii), a look at the proof also shows that it is valid for the limit inferior instead of the limit superior for $T \rightarrow \infty$. Finally, the case of general $A$ is treated as in Step 2 of the proof for Lemma 4.1, above, using projection to the unstable subspace.

We obtain the following characterization of minimal bit rates for stabilization of linear systems.

TheOREM 5.6. Let the assumptions of Corollary 5.4 be satisfied. Then the minimal bit rate for stabilization satisfies

$$
\inf _{\alpha>0} b_{\text {stab }}(\alpha, M)=\log _{2} e \cdot \inf _{\alpha>0} h_{\text {stab }}(\alpha, M)=\log _{2} e \cdot \sum_{\operatorname{Re} \lambda_{i}>0} \operatorname{Re} \lambda_{i} .
$$

Proof. It only remains to show that $b_{\text {stab }}(\alpha, M) \geq \log _{2} e \cdot \sum_{\operatorname{Re} \lambda_{i}>0} \operatorname{Re} \lambda_{i}$ for $\alpha>0$. Consider for $\varepsilon>0$ and $\gamma \in \mathcal{L}_{\varepsilon}$ a $(\gamma, \varepsilon, \alpha, M)$-stabilizing set $\mathcal{R}=\mathcal{R}(\gamma, \varepsilon, \alpha, M)$, hence for all $x \in K$ there is $u \in \mathcal{R}$ with

$$
\|\varphi(t, x, u)\|<\gamma(t)+M e^{-\alpha t}\|x\| \text { for all } t \geq 0 .
$$

By compactness of $K$ one finds $T_{1}>0$ such that for every $x \in K$ there is $u \in \mathcal{R}$ with $\varphi(t, x, u) \in K$ for all $t \geq T_{1}$. Now for $\varepsilon>0$ and all $T>T_{0} \geq T_{1}$ the set of controls $\left\{u_{\mid[0, T]} \mid u \in \mathcal{R}\right\}$ is obviously $\left(T, T_{0}, \varepsilon, K\right)$-spanning. Hence $r_{\text {as }}\left(T, T_{0}, \varepsilon, K\right) \leq \# \mathcal{R}_{T}$. Taking the logarithm, dividing by $T$, and taking the limit for $T \rightarrow \infty$ one finds for every $\varepsilon>0$ and $T_{0} \geq T_{1}$

$$
\varliminf_{T \rightarrow \infty} \frac{1}{T} \ln r_{\mathrm{as}}\left(T, T_{0}, \varepsilon, K\right) \leq \underline{\lim }_{T \rightarrow \infty} \frac{1}{T} \ln \# \mathcal{R}(\gamma, \varepsilon, \alpha, M, K) .
$$

Taking the limit for $T_{0} \rightarrow \infty$ and then the limit for $\varepsilon \searrow 0$, one obtains

$$
\begin{aligned}
h_{\mathrm{as}}(K) & =\lim _{\varepsilon \searrow 0} \lim _{T_{0} \rightarrow \infty} \underline{\lim }_{T \rightarrow \infty} \frac{1}{T} \ln r_{\mathrm{as}}\left(T, T_{0}, \varepsilon, K\right) \\
& \leq \lim _{\varepsilon \searrow 0} \varliminf_{T \rightarrow \infty} \frac{1}{T} \ln \# \mathcal{R}(\gamma, \varepsilon, \alpha, M, K) \\
& =\left[\log _{2} e\right]^{-1} \cdot b_{\mathrm{stab}}(\alpha, M) .
\end{aligned}
$$

By Proposition 5.5 the assertion follows.

Acknowledgments. Thanks are due to Claudio De Persis, Christoph Kawan, Iven Mareels, and Girish Nair for stimulating discussions. Furthermore, Lothar Litz asked a question on an earlier version of this paper leading to Theorem 4.2, and Mario Sigalotti helped with Example 2.1.

\section{REFERENCES}

[1] R. Bowen, Entropy for group endomorphisms and homogeneous spaces, Trans. Amer. Math. Soc., 153 (1971), pp. 401-414.

[2] F. Colonius And C. KaWAn, Invariance entropy for control systems, SIAM J. Control Optim., 48 (2009), pp. 1701-1721.

[3] F. Colonius and C. Kawan, Invariance entropy for outputs, Math. Control Signals Systems, 22 (2011), pp. 203-227.

[4] D. Delchamps, Stabilizing a linear system with quantized state feedback, IEEE Trans. Automat. Control, 35 (1990), pp. 916-924. 
[5] J.-C. Delvenne, An optimal quantized feedback strategy for scalar linear systems IEEE Trans. Automat. Control, 51 (2006), pp. 298-303.

[6] C. DE PERsis, n-Bit stabilization of n-dimensional nonlinear systems in feedforward form, IEEE Trans. Automat. Control, 50 (2005), pp. 299-311.

[7] T. Downarowicz, Topological Entropy, Cambridge University Press, Cambridge, UK, 2011.

[8] F. FAGNANI AND S. A. ZAMPIERI, Symbolic approach to performance analysis of quantized feedback systems: The scalar case, SIAM J. Control Optim., 44 (2005), pp. 816-866.

[9] L. Grüne And O. Junge, Gewöhnliche Differentialgleichungen. Eine Einführung aus der Perspektive der dynamischen Systeme, Vieweg und Teubner, 2009.

[10] A. Katok And B. Hasselblatt, Introduction to the Modern Theory of Dynamical Systems, Cambridge University Press, Cambridge, UK, 1995.

[11] C. Kawan, Invariance Entropy of Control Systems, Doctoral thesis, Institut für Mathematik, Universität Augsburg, 2009.

[12] C. Kawan, Upper and lower estimates for invariance entropy, Discrete Contin. Dyn. Syst., 30 (2011), pp. 169-186.

[13] C. KAWAN, Invariance entropy of control sets, SIAM J. Control Optim., 49 (2011) pp. 732-751.

[14] S. Matveev And A. V. Savkin, Estimation and Control over Communication Networks, Birkhäuser, Boston, 2007.

[15] G. NaIr And R. J. Evans, Exponential stabilisability of finite-dimensional linear systems with limited data rates, Automatica, 39 (2003), 585-593.

[16] G. Nair, F. Fagnani, S. Zampieri, and R. J. Evans, Feedback control under data rate constraints: An overview, Proc. IEEE, 95 (2007), pp. 108-137.

[17] G. Nair, R. J. Evans, I. Mareels, and W. Moran, Topological feedback entropy and nonlinear stabilization, IEEE Trans. Automat. Control, 49 (2004), pp. 1585-1597.

[18] C. Robinson, Dynamical Systems: Stability, Symbolic Dynamics, and Chaos, 2nd ed., CRC Press, Boca Raton, FL, 1999.

[19] E. D. Sontag, Mathematical Control Theory, 2nd ed., Springer-Verlag, New York, 1998.

[20] S. Tatikonda And S. Mitter, Control under communication constraints, IEEE Trans. Automat. Control, 50 (2004), pp. 1056-1068.

[21] P. Walters, An Introduction to Ergodic Theory, Springer-Verlag, New York, 1982.

[22] W. S. Wong And R. W. BRockett, Systems with finite communication bandwidth constraintsII: Stabilization with limited information feedback, IEEE Trans. Automat. Control, 44 (1999), pp. 1049-1053.

[23] L. XIE, Topological entropy and data rate for practical stability: A scalar case, Asian J. Control, 11 (2009), pp. 376-385

Copyright (c) by SIAM. Unauthorized reproduction of this article is prohibited. 